\title{
Efeitos Hemodinâmicos e Metabólicos Imediatos Determinados pelas Soluções de Cloreto de Sódio a 7,5\% e de sua Associação ao Dextran 70 a 6\% na Reanimação do Choque Hemorrágico. Estudo Experimental em Cães*
}

\author{
Immediate Hemodynamic and Metabolic Effects of 7.5\% Sodium \\ Chloride and its Association with 6\% Dextran 70 in Hemorragic Shock \\ Resuscitation. An Experimental Study in Dogs
}

José Fernando Amaral Meletti, TSA ${ }^{1}$; José Reinaldo Cerqueira Braz, TSA²; Norma Sueli Pinheiro Módolo, TSA ${ }^{3}$

\section{RESUMO}

Meletti JFA, Braz JRC, Módolo NSP - Efeitos Hemodinâmicos e Metabólicos Imediatos Determinados pelas Soluções de Cloreto de Sódio a $7,5 \%$ e de sua Associação ao Dextran 70 a $6 \%$ na Reanimação do Choque Hemorrágico. Estudo Experimental em Cães.

JUSTIFICATIVA E OBJETIVOS: O dextran associado à solução hipertônica de cloreto sódio a 7,5\% apresenta efeitos hemodinâmicos benéficos no controle prolongado da reanimação no choque hemorrágico. O objetivo deste estudo foi verificar se a associação do dextran à solução de cloreto de sódio a 7,5\% apresentaria vantagens na avaliação imediata dos parâmetros hemodinâmicos e metabólicos na reanimação em modelo de choque hemorrágico controlado em cães.

MÉTODO: Foram estudados 16 cães submetidos à hemorragia controlada até que a pressão arterial média atingisse $40 \mathrm{mmHg}$ e permanecesse assim por até 30 minutos. Estes foram divididos em G1, com administração de $\mathrm{NaCl}$ a $7,5 \%$, e G2, com administração $\mathrm{NaCl}$ a $7,5 \%$ combinada com dextran 70 a $6 \%$, no volume de 4 $\mathrm{mL} . \mathrm{kg}^{-1}$, durante três minutos. Foram avaliados os parâmetros hemodinâmicos e metabólicos. Consideraram-se quatro momentos: M1 - 10 minutos após o preparo cirúrgico, M2 - obtido na metade da fase de choque, M3 - obtido dois minutos após o final da administração das soluções, M4 - 30 minutos após o início da reanimação.

RESULTADOS: Após a reanimação, não houve diferença significativa dos valores da FC, PAM, PCP e IRVS. O G2 apresentou valores maiores do IC em M4. Os valores da $\mathrm{SvO}_{2}$ foram menores no $\mathrm{G} 1$, final do experimento. $A \mathrm{C}_{(\mathrm{a}-\mathrm{v})} \mathrm{O}_{2}$ foi maior no $\mathrm{G} 1$ nos momentos

*Recebido (Received from) do Laboratório Experimental do Departamento de Anestesiologia da Faculdade de Medicina da Universidade do Estado de São Paulo (FMB - UNESP), Botucatu, SP

1. Professor Adjunto da Disciplina de Anestesiologia da Faculdade de Medicina de Jundiai, SP

2. Professor Titular do CET/SBA da FMB - UNESP

3. Professora Adjunta Livre-Docente do CET/SBA da FMB - UNESP

Apresentado (Submitted) em 09 de dezembro de 2005

Aceito (Accepted) para publicação 27 de junho de 2006

Endereço para correspondência (Correspondence to):

Dr. José Fernando Amaral Meletti

Rua João Massagardi Filho, 98

Jardim Santa Tereza

13211-421 Jundiai, SP

E-mail: nmodolo@fmb.unesp.br

(c) Sociedade Brasileira de Anestesiologia, 2006
M3 e M4. Os valores do $\mathrm{VO}_{2}$ aumentaram nos dois grupos em M4 e os valores do lactato plasmático aumentaram progressivamente até M3 e diminuíram em M4. Houve aumento dos valores do $\mathrm{Na}$ plasmático e redução do hematócrito nos dois grupos.

CONCLUSÕES: O G2 mostrou melhor desempenho hemodinâmico principalmente após 30 minutos do início da reanimação. Observou-se, também, maior expansão plasmática e melhor perfusão tecidual na associação do dextran ao $\mathrm{NaCl}$ a $7,5 \%$.

Unitermos: ANIMAIS: cães; CHOQUE: hipovolêmico; VOLEMIA: expansão cristalóide, dextran.

\section{SUMMARY}

Meletti JFA, Braz JRC, Módolo NSP - Immediate Hemodynamic and Metabolic Effects of $7.5 \%$ Sodium Chloride and its Association with 6\% Dextran 70 in Hemorrhagic Shock Resuscitation. An Experimental Study in Dogs.

BACKGROUND AND OBJECTIVES: Dextran associated with $7.5 \%$ sodium chloride has positive hemodynamic effects in the long term control of hemorrhagic shock. The objective of this study was to verify whether the association of dextran with $7.5 \%$ chloride solution would be advantageous in the immediate hemodynamic evaluation of controlled hemorrhagic shock in dogs.

METHODS: This study included 16 dogs submitted to controlled hemorrhage until their mean arterial blood pressure reached 40 $\mathrm{mmHg}$, being maintained at this level for 30 minutes. They were divided in two groups: $\mathrm{G} 1$ received $7.5 \% \mathrm{NaCl}$ and $\mathrm{G} 2$ received $7.5 \%$ $\mathrm{NaCl}$ in 6\% dextran 70; in both groups, $4 \mathrm{~mL}^{\mathrm{kg}}{ }^{-1}$ of the solutions were administered for three minutes. Hemodynamic and metabolic parameters were evaluated in four different phases: $M 1-10$ minutes after preparation for surgery, M2 - obtained in the middle of the shock phase. M3 - two minutes after the administration of the IV solutions, M4 - 30 minutes after the beginning of the resuscitation.

RESULTS: After the resuscitation, there were no significant differences in HR, MAP, PCP, and SVRI. G2 presented the highest $\mathrm{Cl}$ values in M4. G1 showed the smaller $\mathrm{SvO}_{2}$ values at the end of the experiment; and the $\mathrm{C}_{(-\mathrm{a})} \mathrm{O}_{2}$ was higher in $\mathrm{M} 3$ and M4. For both groups, $\mathrm{VO}_{2}$ values increased in $\mathrm{M} 4$, and lactate plasma values increased progressively until M3, decreasing in M4. Both groups also presented increased Na plasma values and decreased hematocrit.

CONCLUSIONS: G2 showed the best hemodynamic performance, especially 30 minutes after the beginning of resuscitation. Plasma expansion and tissue perfusion were also better with $7.5 \% \mathrm{NaCl}$ in dextran.

Key Words: ANIMALS: dogs; SHOCK: hypovolemic; VOLEMIA: crystalloid expansion, dextran. 


\section{INTRODUÇÃO}

A solução hipertônica de cloreto de sódio tem sido utilizada desde o começo do século passado como tratamento inicial ou coadjuvante do choque hemorrágico. Sua primeira utilização foi descrita para tratamento de soldados americanos na Primeira Guerra Mundial ${ }^{1}$. Nos anos 1960, observou-se que a solução de cloreto de sódio, com 1.800 mOsm. $\mathrm{L}^{-1}$, proporcionava recuperação temporária da pressão arterial em pacientes com choque hemorrágico ${ }^{2}$. Tal resultado foi atribuído à expansão volêmica e vasodilatação pré-capilar ${ }^{3,4}$, decorrente da utilização deste tipo de solução. Velasco e col. demonstraram que cães submetidos a choque hemorrágico (perda sangüínea de 40 a $50 \mathrm{~mL} . \mathrm{kg}^{-1}$ ) apresentaram recuperação da pressão arterial média, do débito cardíaco e melhora da sobrevida, quando se administrava solução hipertônica a 7,5\% (2.400 mOsm. $\left.\mathrm{L}^{-1}\right)$ em volume que correspondia a $10 \%$ do total do sangramento. Nos animais tratados com solução fisiológica a 0,9\% não houve melhora hemodinâmica e os cães evoluíram para óbito em todos os casos ${ }^{5}$.

Os efeitos da solução de $\mathrm{NaCl}$ a $7,5 \%$ eram temporários e desapareciam após uma a duas horas do final da infusão 6,7. A associação do dextran 70 a $6 \%$ à solução hipertônica ${ }^{8}$ faria com que a expansão volêmica fosse acelerada e converteria o efeito pressórico passageiro da solução de dextran pura, em aumento efetivo da pressão arterial e do débito cardíaco ${ }^{9,10}$.

Além do dextran, outras soluções colóides foram avaliadas quanto aos aspectos favoráveis dessas associações. A solução de $\mathrm{NaCl}$ a $7,5 \%$ com dextran 70 a $6 \%$ produziu respostas hemodinâmicas significativamente melhores que a solução de $\mathrm{NaCl}$ hipertônica a 7,5\% com hidroxietilamido a $6 \%{ }^{11}$.

A avaliação comparativa da solução hipertônica a 7,5\% e de sua associação com dextran 70 a $6 \%$, no modelo experimental de choque hemorrágico controlado em porcos, foi estudado em alguns parâmetros hemodinâmicos nos intervalos de 5,15 e 30 minutos após o tratamento. Neste estudo, a associação ao dextran apresentou significativa superioridade ${ }^{12}$. Estudos prospectivos com distribuição aleatória avaliaram os efeitos das soluções hipertônicas a 7,5\% simples ou combinadas com dextran 70 a $6 \%$, como primeiro tratamento no choque hemorrágico, em cerca de 1.500 pacientes internados em unidades hospitalares ${ }^{13-16}$. Estes estudos mostraram que a solução hipertônica combinada com dextran eram seguras e livres de efeitos colaterais tóxicos. Não foram encontrados sinais de complicações renais, neurológicas, cardiopulmonares ou sépticas. Seus efeitos mostraram rápido e significativo aumento da pressão arterial, maior sobrevida e ausência de ressangramento nos pacientes ${ }^{17}$. Para elucidar se a solução hipertônica a $7,5 \%$ ou sua associação ao dextran 70 a $6 \%$ apresentaria diferenças nos parâmetros hemodinâmicos e metabólicos na fase imediata da reanimação no modelo experimental de choque hemorrágico controlado em cães foi desenhado o presente estudo, visto que os efeitos benéficos da adição de solução hiperoncótica à solução hipertônica são evidentes no controle prolongado do choque hemorrágico.

\section{MÉTODO}

Após aprovação pela Comissão de Ética em Experimentação Animal da Faculdade de Medicina da UNESP, Campus de Botucatu, foram estudados 16 cães adultos, machos e fêmeas, sem raça definida, com peso corpóreo entre 16 e $24 \mathrm{~kg}$. Foram divididos em dois grupos com oito cães e submetidos a três fases experimentais: nas fases de preparo do animal e experimental do choque, os animais foram tratados com os mesmos fármacos anestésicos e realizados os mesmos procedimentos. $\mathrm{Na}$ fase experimental de reanimação, os grupos foram divididos pelo tipo de solução utilizada na reanimação.

G1: solução hipertônica: solução hiperosmolar de cloreto de sódio a $7,5 \%(\mathrm{SH})-4 \mathrm{~mL} . \mathrm{kg}^{-1}$;

G2: solução hipertônica com dextran: solução hiperosmolar de cloreto de sódio a $7,5 \%$ associada à solução hiperoncótica de dextran 70 a $6 \%$ (SHD) - 4 mL. $\mathrm{kg}^{-1}$.

Após jejum alimentar de 12 horas, os animais foram anestesiados com $30 \mathrm{mg} \cdot \mathrm{kg}^{-1}$ de pentobarbital sódico e 3,6 $\mathrm{mg} \cdot \mathrm{kg}^{-1} \cdot \mathrm{h}^{-1}$ para manutenção da anestesia (em bomba de infusão modelo Anne, Abbot, USA) e colocados sobre a goteira de Claude Bernard, realizando-se a seguir:

1. Intubação traqueal e instalação de ventilação mecânica controlada, com circuito de reinalação, através de respirador modelo 674 do aparelho de anestesia K. Takaoka, modelo 2600. O volume corrente foi de $12 \mathrm{~mL} . \mathrm{kg}^{-1}$, a freqüência respiratória de 16 irpm e o fluxo de oxigênio de 1 L. min $^{-1}$.

2. Dissecção e cateterização das veias femoral, esquerda e direita, para administração de fármacos anestésicos (pentobarbital sódico e cloreto de pancurônio 0,08 mg. $\mathrm{kg}^{-1}$ ) e coleta de amostras sangüíneas, respectivamente.

3. Dissecção e cateterização da artéria femoral esquerda para monitorização da pressão arterial invasiva e da artéria femoral direita para a realização do sangramento.

4. Dissecção da veia jugular externa direita e instalação do cateter de Swan-Ganz.

5. Calibração do monitor Datex Engstron, tipo D-VNC 15-00$02 \mathrm{~s} / \mathrm{n}^{\circ} 763177$.

6. Verificação do correto posicionamento do cateter.

7. Monitorização com eletrocardiograma contínuo na derivação $D_{\|}$, sensor de oxímetro de pulso na língua do animal e termômetro timpânico na orelha esquerda do animal.

8. Execução de laparotomia mediana para realização de esplenectomia com hemostasia rigorosa da ferida cirúrgica.

9. Complementação da dose de cloreto de pancurônio $\left(0,02\right.$ mg. $\left.\mathrm{kg}^{-1}\right)$ e estabilização do animal por 10 minutos.

10. Realização do primeiro momento de estudo (momento controle): coleta de sangue arterial, venoso misto e de- 
terminação dos parâmetros hemodinâmicos (freqüência cardíaca, pressão arterial sistólica, pressão arterial média, pressão arterial pulmonar, pressão venosa central, pressão capilar pulmonar, débito cardíaco, índice cardíaco, índice sistólico, índice de resistência vascular sistêmica, índice de resistência vascular pulmonar e índice do trabalho sistólico do ventrículo esquerdo).

11. Realização do sangramento dois minutos após o final do primeiro momento.

12. Sangramento de $10 \%$ do volume calculado para volemia pelo cateter da artéria femoral direita e observação dos parâmetros hemodinâmicos por cinco minutos.

13. Retirada de mais $10 \%$ do volume de sangue calculado para o animal pelo cateter da artéria femoral direita e observação dos parâmetros hemodinâmicos para verificação da necessidade de nova hemorragia por cinco minutos.

14. Interrupção do sangramento quando a pressão arterial média alcançasse valores de aproximadamente 40 $\mathrm{mmHg}$ por um período de cinco minutos.

15. Estabilização do animal por 30 minutos.

16. Coleta de sangue arterial, venoso misto e determinação dos mesmos parâmetros hemodinâmicos na metade do período de estabilização (M2).

17. Administração de SH (G1) ou SHD (G2) no volume de 4 $\mathrm{mL} . \mathrm{kg}^{-1}$ durante três minutos.

18. Coletas de sangue arterial, venoso misto e determinação dos parâmetros hemodinâmicos dois minutos, após o final da infusão da solução hipertônica (M3).
19. Estabilização do animal por 25 minutos.

20. Coletas de sangue arterial, venoso misto e determinação dos parâmetros hemodinâmicos 30 minutos após o início da administração da solução hipertônica (M4).

21. Final do experimento.

\section{Parâmetros Estudados}

1. Hemodinâmicos: freqüência cardíaca (FC - bat. $\left.\min ^{-1}\right)$, pressão arterial média (PAM - mmHg), pressão capilar pulmonar (PCP - mmHg), índice cardíaco (IC - L. $\mathrm{min}^{-1}$. $\mathrm{m}^{-2}$ ), índice de resistência vascular sistêmica (IRVS dinas.s. $\left.\mathrm{cm}^{-5} \cdot \mathrm{m}^{-2}\right)$.

2. Oxigenação tecidual e metabólicos: saturação venosa mista $\left(\mathrm{SvO}_{2}-\%\right)$, diferença arteriovenosa de oxigênio $\left(\mathrm{C}(\mathrm{a}-\mathrm{v}) \mathrm{O}_{2}-\mathrm{mL} .100 \mathrm{~mL}^{-1}\right)$, transporte de oxigênio $\left(\mathrm{DO}_{2}-\right.$ $\left.\mathrm{mL} \cdot \mathrm{min}^{-1}\right)$, consumo de oxigênio $\left(\mathrm{VO}_{2}-\mathrm{mL} \cdot \mathrm{min}^{-1}\right)$, lactato plasmático (Lactato - mEq. $\mathrm{L}^{-1}$ ).

3. Equilíbrio ácido-base: $\mathrm{pH}$, pressão parcial de gás carbônico $\left(\mathrm{PaCO}_{2}-\mathrm{mmHg}\right)$.

4. Hematológicos: sódio plasmático $\left(\mathrm{Na}-\mathrm{mEq} \cdot \mathrm{L}^{-1}\right)$, Hematócrito $(\mathrm{Ht}-\%)$.

Os dados relativos aos parâmetros foram distribuídos em quatro momentos: M1 - obtido após 10 minutos de estabilização do preparo cirúrgico, considerado como parâmetro inicial ou controle; M2 - após a fase de choque, 15 minutos depois do início do período de estabilização de 30 minutos; M3 - dois minutos após o final da infusão da solução hipertônica dos grupos selecionados; M4 - obtido 30 minutos

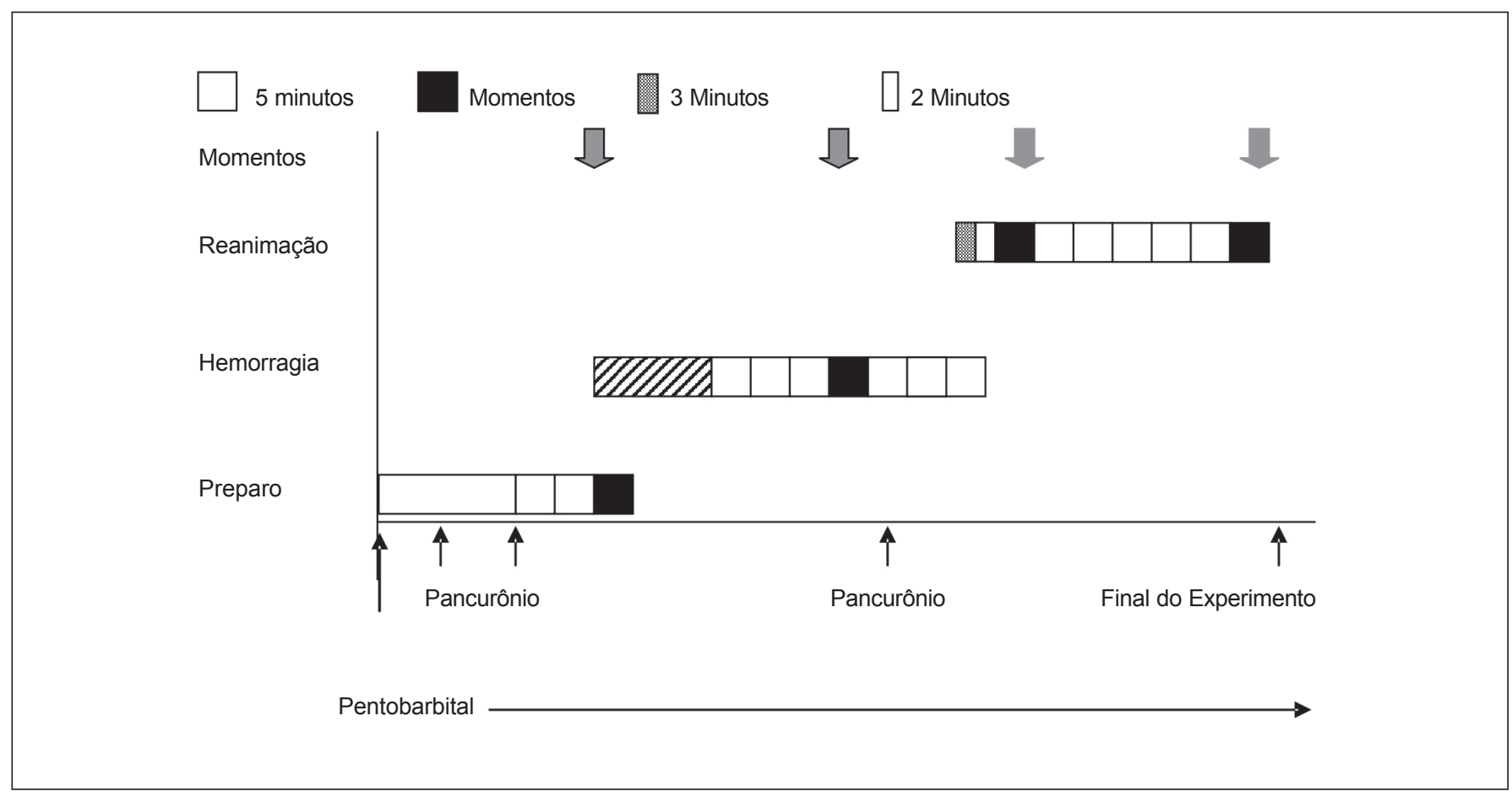

Figura 1 - Seqüência Experimental 
após o início da reanimação com solução hipertônica dos grupos selecionados.

\section{Análise Estatística}

Para o peso, a altura e o volume de sangramento, a análise estatística foi avaliada em um momento, a comparação entre os grupos foi efetuada pelo teste $t$ para duas amostras independentes, com o cálculo de $t$ e $\mathrm{p}^{18}$.

Para verificação das variáveis ao longo do tempo foi utilizada Análise de Perfil ${ }^{19}$. Foram calculados $\mathrm{F}$ e $\mathrm{p}$ em cada hipótese. Em todas as análises efetuadas os valores calculados foram considerados significativos quando $p<$ 0,05 . Quando $0,05<p<0,10$, foi referida tendência significativa ( $p$ é a probabilidade de conclusão errônea pela significância).

\section{RESULTADOS}

Houve similaridade (teste $t$ ) nas variáveis peso, comprimento, superfície corpórea e volume de sangramento entre os animais dos dois grupos experimentais (Tabela I).

Nos dois grupos, os valores da FC foram menores no final do sangramento e no momento inicial da reanimação, retor- nando a valores iniciais (controle) no final do estudo. Em ambos os grupos, foram encontrados valores da PAM aumentados após a reanimação (M3 e M4); entretanto, esses valores não retornaram aos valores iniciais (M1). Os valores da PCP no G2 apresentaram-se menores com relação ao G1 no segundo momento do experimento; apesar disto, os valores encontrados nos momentos referentes à reanimação foram iguais nos dois grupos estudados. Apesar de os grupos apresentarem perfis similares, no quarto momento do $\mathrm{G} 2$ os valores do IC foram discretamente maiores que no G1. Os valores do IRVS encontravam-se aumentados no momento-controle nos dois grupos, permaneceram elevados após a hemorragia e apresentaram diminuição significativa de seus valores no momento inicial da reanimação; entretanto, os valores retornaram aos encontrados no momento controle no final do estudo (Tabela II).

Os valores da $\mathrm{SvO}_{2}$ apresentaram perfil não-similar na interação entre grupos e momentos. No quarto momento do G2 foram encontrados valores significativamente maiores deste índice em relação ao G1. Com relação à diferença arteriovenosa de oxigênio, observou-se que o G1 apresentou valores significativamente maiores nos momentos correspondentes à reanimação volêmica. No momento-controle, os valores do $\mathrm{DO}_{2}$ estavam elevados, nos dois grupos. $\mathrm{Na}$

Tabela I - Peso, Comprimento, Superfície Corpórea e Sangramento

\begin{tabular}{lccccc}
\hline Variáveis & $\mathrm{G} 1{ }^{*}$ & $\mathrm{G} 2{ }^{*}$ & Valor de $\mathrm{p}$ & Valor de $t$ & Comentário \\
\hline Peso $(\mathrm{kg})$ & $20,5 \pm 2,67$ & $20,5 \pm 3,07$ & $>1,00$ & 0,0 & $\mathrm{G} 1=\mathrm{G} 2$ \\
Comprimento $(\mathrm{cm})$ & $105,5 \pm 5,32$ & $101 \pm 8,19$ & $>0,1$ & 1,18 & $\mathrm{G} 1=\mathrm{G} 2$ \\
Superfície corpórea $\left(\mathrm{m}^{2}\right)$ & $0,75 \pm 0,062$ & $0,73 \pm 0,086$ & $>0,1$ & 0,57 & $\mathrm{G} 1=\mathrm{G} 2$ \\
Sangramento $(\mathrm{mL})$ & $683,8 \pm 184,3$ & $680,6 \pm 157,5$ & $>0,5$ & 0,04 & $\mathrm{G} 1=\mathrm{G} 2$ \\
\hline
\end{tabular}

* Valores expressos em média \pm DP

Tabela II - Parâmetros Hemodinâmicos

\begin{tabular}{|c|c|c|c|c|c|c|}
\hline \multirow[t]{2}{*}{ Parâmetros } & \multirow[t]{2}{*}{ Grupos } & \multicolumn{4}{|c|}{ Momentos } & \multirow[t]{2}{*}{ Análise Estatística } \\
\hline & & M1 & M2 & M3 & M4 & \\
\hline FC $\left(\right.$ bat. $\left.\mathrm{min}^{-1}\right)$ & G1 & $169,25 \pm 26,57$ & $152 \pm 32,54$ & $154,5 \pm 29,74$ & $171 \pm 35,71$ & $\mathrm{G} 1=\mathrm{G} 2(p=0,5)$ \\
\hline \multirow[t]{2}{*}{ PAM $(\mathrm{mmHg})$} & $\mathrm{G} 1$ & $131,25 \pm 15,81$ & $43,00 \pm 12,57$ & $78,38 \pm 11,49$ & $92,5 \pm 16,06$ & $\mathrm{G} 1=\mathrm{G} 2(\mathrm{p}=0,5)$ \\
\hline & G2 & $129,62 \pm 9,18$ & $40,62 \pm 13,46$ & $79,75 \pm 14,44$ & $91,88 \pm 11,69$ & $\mathrm{M} 1>\mathrm{M} 4>\mathrm{M} 3$ > M2 \\
\hline \multirow[t]{2}{*}{ IC $\left(\mathrm{L} \cdot \mathrm{min}^{-1} \cdot \mathrm{m}^{-2}\right)$} & $\mathrm{G} 1$ & $5,06 \pm 1,27$ & $1,7 \pm 0,57$ & $4,35 \pm 0,8$ & $3,4 \pm 0,69$ & Tendência de G1 < G2 \\
\hline & G2 & $5,41 \pm 1,21$ & $1,43 \pm 0,5$ & $74,91 \pm 0,94$ & $4,3 \pm 1,16$ & $\begin{array}{c}(0,05<p<0,10) \text { em M4 } \\
(\mathrm{M} 1=\mathrm{M} 3)>\mathrm{M} 4>\mathrm{M} 2\end{array}$ \\
\hline IRVS & G1 & $2159,25 \pm 521,05$ & $2170,25 \pm 771,04$ & $1522,62 \pm 477,61$ & $2260,12 \pm 610,17$ & $G 1=G 2(p>0,5)$ \\
\hline$\left(\right.$ dina.s. $\left.\mathrm{cm}^{-5} \cdot \mathrm{m}^{-2}\right)$ & G2 & $1980,25 \pm 430,5$ & $2508,62 \pm 863,76$ & $1268,87 \pm 229,7$ & $1902,37 \pm 533,24$ & $(M 1=M 2=M 4)>M 3$ \\
\hline
\end{tabular}

Freqüência Cardíaca (FC), Pressão Arterial Média (PAM), Pressão Capilar Pulmonar (PCP), Índice Cardíaco (IC), Índice de Resistência Vascular Sistêmica (IRVS). 
fase de choque os valores apresentaram reduções drásticas e voltaram a aumentar após a reanimação volêmica, porém sem atingir os índices encontrados no momentocontrole. O G1 apresentou maiores valores do $\mathrm{VO}_{2}$ do que o G2. O comportamento do lactato plasmático foi de elevação gradativa de seus valores até o primeiro momento da reanimação, quando no final do experimento verificou-se diminuição considerável deste índice a valores correspondentes ao período de choque hipovolêmico (Tabela III).

Os valores de $\mathrm{pH}$ ficaram inalterados com o choque hemorrágico, e diminuíram após a reanimação com as so- luções hipertônicas. Após a reanimação, os valores da pressão parcial de gás carbônico elevaram-se de forma acentuada em ambos os grupos (Tabela IV).

Nos dois grupos experimentais foram observados aumentos acentuados e iguais dos valores do sódio plasmático após a reanimação volêmica. Os valores do hematócrito apresentaram reduções significativas após a hemorragia e o momento imediato da reanimação, vindo a estabilizar seus valores no momento que seguiu a administração dos dois tipos de solução hipertônica nos grupos experimentais estudados (Tabela V).

Tabela III - Parâmetros da Oxigenação Tecidual e Metabólicos

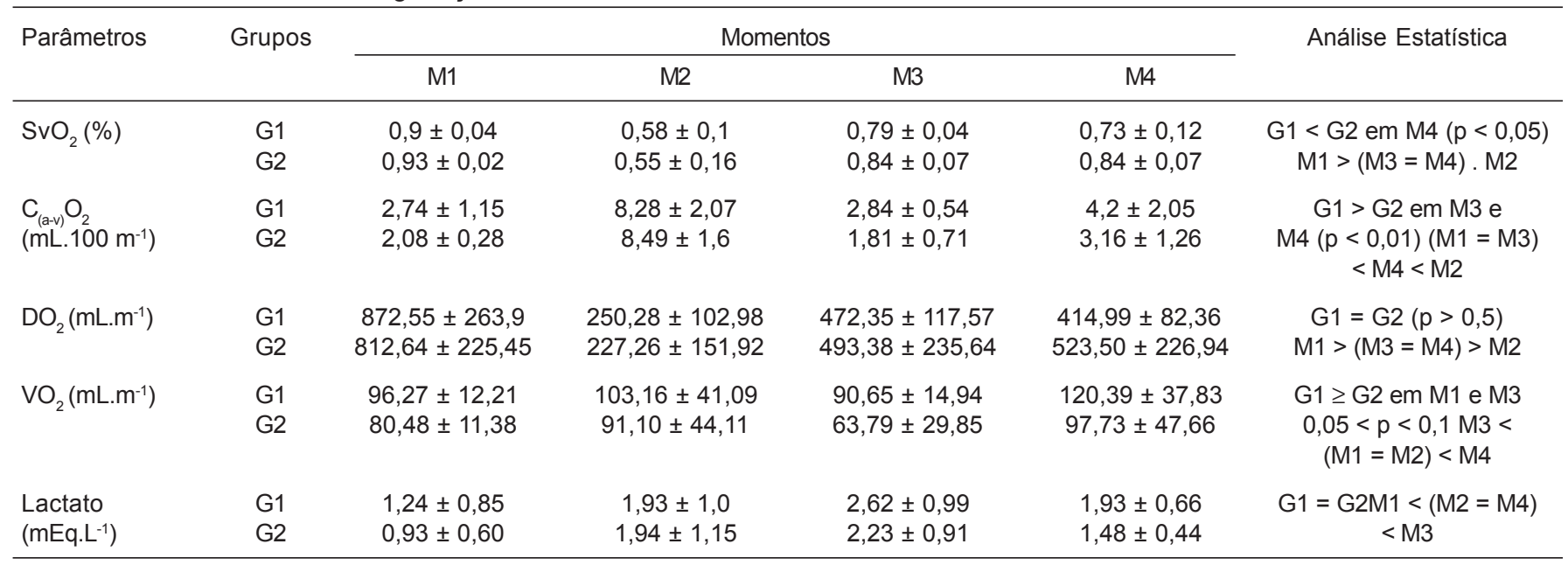

Saturação Venosa Mista $\left(\mathrm{SvO}_{2}\right)$, Diferença Arteriovenosa de Oxigênio $\left(\mathrm{C}_{(a-v)} \mathrm{O}_{2}\right)$, Transporte de Oxigênio $\left(\mathrm{DO}_{2}\right)$, Consumo de Oxigênio $\left(\mathrm{VO}_{2}\right)$ e Lactato Plasmático (lactato)

Tabela IV - Parâmetros do Equilíbrio Ácido-Base

\begin{tabular}{lcccccc}
\hline \multirow{2}{*}{ Parâmetros } & \multirow{2}{*}{ Grupos } & \multicolumn{2}{c}{ Momentos } & Análise Estatística \\
\cline { 3 - 6 } & & $\mathrm{M} 1$ & $\mathrm{M} 2$ & $\mathrm{M} 3$ & $\mathrm{M} 4$ \\
\hline $\mathrm{nH}$ & $\mathrm{G} 1$ & $7,28 \pm 0,06$ & $7,24 \pm 0,07$ & $7,13 \pm 0,11$ & $7,17 \pm 0,08$ & $\mathrm{G} 1=\mathrm{G} 2$ \\
& $\mathrm{G} 2$ & $7,32 \pm 0,07$ & $7,22 \pm 0,1$ & $7,10 \pm 0,11$ & $7,5 \pm 0,09$ & $(\mathrm{M} 1=\mathrm{M} 2)>(\mathrm{M} 3=\mathrm{M} 4)$ \\
$\mathrm{PaCO}_{2}$ & $\mathrm{G} 1$ & $49,75 \pm 11,69$ & $45,12 \pm 10,26$ & $66,66 \pm 15,64$ & $64,83 \pm 16,83$ & $\mathrm{G} 1=\mathrm{G} 2$ \\
& $\mathrm{G} 2$ & $49,36 \pm 13,86$ & $50,55 \pm 11,43$ & $71,48 \pm 12,11$ & $61,87 \pm 8,4$ & $(\mathrm{M} 1=\mathrm{M} 2)<(\mathrm{M} 3=\mathrm{M} 4)$ \\
\hline
\end{tabular}

pH do Sangue Arterial $(\mathrm{pH})$, Pressão Parcial de Gás Carbônico $\left(\mathrm{PaCO}_{2}\right)$.

Tabela V - Parâmetros Hematológicos

\begin{tabular}{|c|c|c|c|c|c|c|}
\hline \multirow[t]{2}{*}{ Parâmetros } & \multirow[t]{2}{*}{ Grupos } & \multicolumn{4}{|c|}{ Momentos } & \multirow[t]{2}{*}{ Análise Estatística } \\
\hline & & M1 & M2 & M3 & M4 & \\
\hline Sódio plasmático & G1 & $145,5 \pm 5,04$ & $140,5 \pm 8,66$ & $153,5 \pm 8,89$ & $148,5 \pm 4,75$ & $\mathrm{G} 1>\mathrm{G} 2 \mathrm{em} \mathrm{M} 1$ \\
\hline
\end{tabular}




\section{DISCUSSÃO}

A técnica anestésica empregada é recomendada pela maioria dos autores para esse modelo experimental, a fim de que os animais apresentem plano anestésico-cirúrgico adequado em poucos minutos $5,20-23$. Foi realizada infusão contínua de pentobarbital sódico $\left(3,6 \mathrm{mg} \cdot \mathrm{kg}^{-1} \cdot \mathrm{h}^{-1}\right)$ para que a concentração plasmática do fármaco não se alterasse e fosse suficiente para que o cão permanecesse anestesiado durante todo o período experimental ${ }^{24-26}$.

O modelo experimental de choque hemorrágico utilizando animais tem sido freqüentemente estudado pelo modelo de Wiggers, $1942{ }^{27}$, em que o animal é induzido a permanecer num profundo estado de choque através do sangramento, mantendo-se a pressão arterial média entre 35 e 40 $\mathrm{mmHg}$. Vários pesquisadores modificaram esse modelo de diversas maneiras, com a finalidade de atender aos objetivos propostos em seus trabalhos ${ }^{28-30}$.

A velocidade de injeção da solução hipertônica ficou estabelecida em três minutos, em conformidade com outros trabalhos ${ }^{8,9,13,31}$, sendo este um tempo adequado para não causar lesão histológica na parede vascular ${ }^{31}$. O volume de solução infundido para cada grupo foi de $4 \mathrm{~mL} . \mathrm{kg}^{-1}$. Este volume tem sido proposto na maioria dos trabalhos pesquisados, tanto experimentais quanto os realizados em seres humanos $5,7,8,11,32$.

Observou-se neste estudo que, na fase do choque hemorrágico, a freqüência cardíaca apresentou diminuição significativa, que pode ser explicada pelo reflexo de Baindridge, em que se observou bradicardia na diminuição do retorno venoso, pela maior sensibilidade maior dos barorreceptores venosos com relação aos arteriais ${ }^{33,34}$. Portanto, nesta situação, a redução significativa do retorno venoso pode ter diminuído a FC.

No momento da reanimação com ambas as soluções empregadas neste estudo, a FC não apresentou alterações, situação também observada em outros estudos em que se optou pela infusão rápida de solução hipertônica ${ }^{12,35}$. Após 30 minutos do início da administração das soluções a osmolaridade plasmática elevou-se pelo acréscimo de maior concentração de sódio, determinando melhora do desempenho cardíaco e aumento da FC com relação aos valores encontrados no momento controle, pré-choque ${ }^{23,36}$.

A solução hipertônica simples apresenta efeito temporário de melhora das condições hemodinâmicas, a menos que se adicione solução colóide à fórmula da solução hiperosmolar. Enquanto a hipertonicidade da solução seria a responsável pela retirada de líquido do espaço intracelular, a solução colóide hiperoncótica manteria este líquido no espaço intravascular. Os efeitos fisiológicos dos pequenos volumes de solução hipertônica estão associados ao aumento da pressão arterial média, do débito cardíaco e da expansão plasmática. As soluções também provocam o aumento do consumo de oxigênio, dilatam os esfíncteres précapilares, aumentam a contratilidade cardíaca, a diurese e a natriurese, restauram o potencial de membrana e diminuem a necessidade de volume para restaurar a volemia no controle prolongado do choque ${ }^{11}$.

Neste trabalho, ambas soluções apresentaram desempenho hemodinâmico semelhantes no segundo minuto após o final da administração, portanto, mais precoce que os outros trabalhos pesquisados na literatura. Entretanto no seguimento da reanimação e até 30 minutos após o início desta, a superioridade da solução combinada com dextran é incontestável, sobretudo quando foi analisado o índice cardíaco nos dois grupos.

Os efeitos das soluções hipertônicas a 7,5\% sobre a pressão arterial, o débito cardíaco e o índice sistólico são quase que instantâneos e duram de 45 minutos na solução hipertônica simples e até duas a quatro horas, quando esta for combinada ao dextran 70 a $6 \%$. $O$ aumento da contratilidade cardíaca independe da pré-carga ${ }^{17}$.

A causa mais provável da vasodilatação é o efeito da osmolaridade na parede arteriolar ou a existência de um reflexo de vasodilatação, desencadeado por receptores pulmonares sensíveis à osmolaridade plasmática aumentada. A característica de vasodilatação dos territórios vasculares, desencadeada pelas soluções hipertônicas, diminui a resistência vascular sistêmica e a resistência vascular pulmonar de forma direta, independentemente da inervação local, sendo observada na grande maioria dos territórios vasculares ${ }^{17}$. Quando foram analisados os resultados dos parâmetros hemodinâmicos, verificou-se que as soluções hiperosmolares a $7,5 \%$ produziram efeitos cardiovasculares imediatos e peculiares. Basicamente ocorreu importante vasodilatação pré-capilar, com diminuição da resistência vascular sistêmica, melhora na contratilidade miocárdica, aumento da pressão arterial média e da pressão arterial pulmonar e elevação dos índices de pré-carga.

A adição da solução hiperoncótica de dextran 70 a $6 \%$ à solução de $\mathrm{NaCl}$ a $7,5 \%$, fez com que, no momento imediato da reanimação (M3), não houvesse diferença significativa no comportamento hemodinâmico dos cães neste experimento. Ao analisarem-se os resultados do momento referente a 30 minutos do início da reanimação, percebeu-se que a SHD apresentou melhor desempenho cardiovascular, sobretudo no parâmetro índice cardíaco.

Em condições fisiológicas normais, o consumo de oxigênio é determinado pela necessidade metabólica tecidual, independente da oferta de oxigênio. É o que ocorre em situações de metabolismo aumentado ou de baixa perfusão tecidual, a extração de oxigênio, que normalmente é de $25 \%$, passa a ser de $75 \%$ a $80 \%$. Nessas condições, ocorrem drásticas reduções do conteúdo de oxigênio medido no compartimento central ${ }^{37}$.

Nesta pesquisa observou-se diminuição de cerca de $38 \%$ da saturação venosa mista quando houve perda de cerca $46 \%$ da volemia dos cães, na fase de choque hemorrágico, tal resultado foi semelhante ao relatado na literatura ${ }^{28,38}$. 
Com relação à saturação venosa mista, os dois grupos, neste experimento, tiveram o mesmo comportamento no momento imediato da reanimação; entretanto, no final do experimento, os valores da saturação venosa mista do grupo da SHD mantiveram-se inalterados e os valores da $\mathrm{SvO}_{2}$ apresentaram reduções significativas no grupo da $\mathrm{SH}$. Tais resultados mostraram que a associação do dextran 70 à solução de $\mathrm{NaCl}$ a $7,5 \%$ foi superior à solução hipertônica com relação à saturação venosa mista de oxigênio, nos 30 minutos iniciais da reanimação. Os valores menores da $\mathrm{SvO}_{2}$ no grupo de $\mathrm{SH}$, demonstraram que o fluxo sangüíneo na microcirculação estava mais reduzido neste grupo, levando à maior extração de oxigênio com relação ao grupo de solução hipertônica associado ao dextran 70 .

$\mathrm{O}$ transporte de oxigênio $\left(\mathrm{DO}_{2}\right)$ é o produto do conteúdo arterial de oxigênio $\left(\mathrm{CaO}_{2}\right)$ pelo débito cardíaco (DC). O decréscimo do conteúdo arterial de oxigênio, por sua vez, depende da concentração e saturação de oxigênio pela hemoglobina. Assim, uma diminuição no $\mathrm{DO}_{2}$ pode ser corrigida com transfusão sangüínea ou aumento no débito cardíaco. O débito cardíaco tende a estar aumentado em situações em que existe elevado consumo de oxigênio, como no trauma ${ }^{39}$.

$\mathrm{O}$ consumo de oxigênio $\left(\mathrm{VO}_{2}\right)$ é o produto do conteúdo venoso de oxigênio pelo débito cardíaco. A redução ou o inadequado consumo de oxigênio é o denominador comum em todas as síndromes de choque. Mais ainda, o $\mathrm{VO}_{2}$ parece estar relacionado com a sobrevida dos pacientes com choque hemorrágico ${ }^{40}$.

O índice de diferença arteriovenosa do conteúdo de oxigênio $\left(\mathrm{C}_{(\mathrm{a}-\mathrm{v})} \mathrm{O}_{2}\right)$, representa a reação entre o consumo de oxigênio e o débito cardíaco. Por este motivo, ele representa intrinsecamente um índice de adequação da função hemodinâmica, sua elevação vai representar, geralmente, estados cardiocirculatórios hipodinâmicos, tanto por descompensação cardíaca, como por hipovolemia ${ }^{41}$.

Com a perda sangüínea de aproximadamente $34 \mathrm{~mL} . \mathrm{kg}^{-1}$, observou-se nos dois grupos, neste experimento, diminuição dos valores do transporte de oxigênio e aumento dos valores da diferença arteriovenosa de oxigênio, visto que o consumo de oxigênio apresentou-se inalterado com a hemorragia. O consumo de oxigênio deve ser encarado como um índice de prognóstico no choque hemorrágico, quando há redução de seus valores, instala-se a fase de choque irreversível ${ }^{3}$. Hannon e col. ${ }^{42}$ avaliaram a relação entre o transporte de oxigênio e a demanda de $\mathrm{O}_{2}$ durante a hemorragia de cerca de $37 \mathrm{~mL} . \mathrm{kg}^{-1} \mathrm{em}$ uma hora e na subseqüente reanimação com $\mathrm{NaCl}$ a $7,5 \%$ com dextran 70 a $6 \%$ na dose de $4 \mathrm{~mL} . \mathrm{kg}$ ${ }^{1}$, no modelo experimental de choque em porcos. Na fase de choque foi encontrado pequeno aumento dos valores do $\mathrm{VO}_{2}$, aumento acentuado dos valores do lactato plasmático, redução significativa dos valores do $\mathrm{DO}_{2}$ e uma aparente duplicação numérica dos valores da demanda de oxigênio. A diferença entre os valores encontrados do transporte de oxigênio e a demanda de oxigênio foi diminuída pela reanimação volêmica com a solução hipertônica, primariamente por supressão da demanda metabólica e secundariamente pelo aumento do $\mathrm{DO}_{2}$. O desempenho hemodinâmico e metabólico das soluções de SH e SHD em 5, 15 e 30 minutos após a reanimação de porcos em choque hemorrágico mostrou que os valores do transporte de oxigênio foram significativamente maiores no grupo com dextran em todos os momentos estudados.

Clinicamente, o aumento do lactato plasmático correlaciona-se muito bem com a deficiência de oxigênio e com a respiração anaeróbica. Em humanos, níveis de lactato superiores a $2 \mathrm{mEq} . \mathrm{L}^{-1}$ estão associados ao aumento de mortalidade no choque, e valores superiores a 3 e $4 \mathrm{mEq} . \mathrm{L}^{-1}$ evidenciam significativa hipoperfusão.

Em condições aeróbicas, o metabolismo dos hidratos de carbono segue a via do ciclo de Krebs; assim, a oxidação seqüencial de uma molécula de glicose resulta na formação de 38 moléculas de ATP. Quando o suprimento de oxigênio é insuficiente, a seqüência habitual é interrompida. O piruvato é convertido em lactato e o rendimento energético limita-se à produção de apenas duas moléculas de ATP. O lactato formado em condições anaeróbicas acumula-se no organismo, pois só é utilizado em número reduzido de processos depurativos que ocorrem basicamente nos hepatócitos ${ }^{37}$. Strecker e col. ${ }^{43}$ pesquisaram a eficácia do dextran a $6 \%$ ou do hidroxietilamido a $6 \%$ na associação com a solução hipertônica a $7,5 \%$ na reanimação do choque hemorrágico em coelhos. Em ambos os grupos os valores da diferença arteriovenosa de oxigênio retornaram aos parâmetros iniciais (préchoque) em dois minutos após a reanimação, exatamente como se verificou nesta pesquisa, porém, nos momentos de 15 e 60 minutos após a reanimação, Strecker e col. verificaram aumento significativo dos valores deste índice no grupo com hidroxietilamido. Com relação ao lactato sérico e o excesso de base, este estudo demonstrou diminuição imediata dos níveis, em ambos os grupos, porém menos acentuado no grupo com dextran. Os valores do lactato e do BE apresentaram redução significativa e foi verificado maior diminuição dos valores no grupo com dextran com relação ao hidroxietilamido 60 minutos após a reanimação.

A redução do volume circulante determinou a redução da função cardiovascular no estudo de Strecker e col. Esta, por sua vez, reduziu consideravelmente a $\mathrm{TEO}_{2}$ na fase de choque hemorrágico. Após a reanimação com as duas soluções empregadas no estudo, o grupo da solução hipertônica com dextran apresentou valores maiores do $\mathrm{DC}$ e do $\mathrm{DO}_{2}$, desta forma, se observou valores menores na diferença arteriovenosa de oxigênio no SHD com relação ao grupo composto pela solução com hidroxietilamido. Os autores explicaram que a maior elevação dos valores do lactato, nos momentos iniciais da reanimação no grupo da SHD, foi decorrente do aumento da perfusão tecidual em áreas com maior concentração de lactato, resultante do choque.

Nesta pesquisa observou-se que os valores da $\mathrm{C}_{(\mathrm{a}-\mathrm{v})} \mathrm{O}_{2}$ foram maiores no grupo com solução hipertônica simples nos dois momentos da reanimação e os valores da $\mathrm{TEO}_{2}$ exibiram 
diferença estatística significativa, sendo maiores no $\mathrm{G} 1,30$ minutos após o início da administração da solução. Tal resultado indicou que o G1 apresentou maior grau de hipofluxo sistêmico com relação ao G2, proporcionando, desta forma, maior extração de oxigênio pelas células e menor saturação venosa mista encontrada no G1 no final do estudo.

Neste experimento verificaram-se diferenças significativas dos valores de lactato plasmático nos grupos estudados e somente observou-se diminuição dos seus valores no final do estudo.

Chiara e col. ${ }^{44}$ também compararam a solução de $\mathrm{NaCl}$ a $7,5 \%$ e a sua combinação com dextran $70 \mathrm{em}$ modelo experimental de choque hemorrágico em porcos. Neste estudo concluíram que as soluções não apresentaram diferenças estatísticas nos momentos iniciais da reanimação quando avaliadas pelo $\mathrm{DO}_{2}, \mathrm{VO}_{2}$ e $\mathrm{TEO}_{2}$. Somente uma hora após a administração das soluções, o grupo da SHD apresentou aumento significativo desses índices com relação ao grupo da $\mathrm{SH}$.

Após a administração de solução hipertônica de cloreto de sódio (1.800 mOsm. $\mathrm{L}^{-1}$ ) houve diminuição no $\mathrm{pH}$ e aumento da pressão parcial de gás carbônico ${ }^{2}$. Fenômeno semelhante foi encontrado após a administração de solução de $\mathrm{NaCl}$ a $7,5 \%{ }^{5}$.

$\mathrm{O} \mathrm{pH}$ arterial alterou-se de 7,34 para 7,26 após a retirada de cerca de $44 \%$ da volemia estimada para cada cão ${ }^{45}$. Na presente pesquisa, a redução do $\mathrm{pH}$ foi de em torno de 7,30 para 7,23 em ambos os grupos, não havendo diferenças significativas na variação do $\mathrm{pH}$ com a retirada de cerca de $46 \%$ da volemia calculada para cada cão.

Neste trabalho, após a reanimação volêmica, ambos os grupos também apresentaram comportamentos iguais. $\mathrm{O} \mathrm{pH}$ e a pressão parcial de gás carbônico apresentaram elevações significativas e sustentadas, tanto em M3 quanto em M4, em concordância com outras pesquisas ${ }^{42,43}$.

A administração da solução hipertônica a 7,5\% associada ou não ao dextran determinou elevação dos valores da $\mathrm{PaCO}_{2}$ e diminuição do $\mathrm{pH}$ arterial de forma significativa logo após a reanimação de choque hemorrágico. Tal efeito deveu-se a breve acidemia causada primariamente pela hipercloremia, hipocalemia e acidose metabólica com anion gap normal secundário à acidose respiratória causada pela elevação da $\mathrm{PaCO}_{2}{ }^{46,47}$.

Neste trabalho a piora da acidose nos momentos iniciais da reanimação ocorreu provavelmente em decorrência da presumida hipercloremia resultante da administração da solução hipertônica, não havendo tempo suficiente para a sua recuperação. $O$ aumento súbito da pressão parcial de gás carbônico indicou melhora na perfusão tecidual nos dois grupos, mas também contribuiu bastante para a manutenção da acidemia registrada na reanimação.

A substituição do cloro pelo acetato na fórmula da solução hipertônica, com o objetivo de proporcionar reanimação isoclorêmica, mostrou-se inadequada. Apesar de não ocorrer elevação do cloro plasmático e apresentar rápida correção da acidose sangüínea, sua administração determinou insatisfatório desempenho cardiovascular e metabólico comparada às soluções tradicionais ${ }^{48}$.

O comportamento do hematócrito apresentou redução dos valores imediatamente após a reanimação. Tal fenômeno foi explicado devido à expansão aguda provocada pela solução de $\mathrm{NaCl}$ a $7,5 \%$ no compartimento intravascular e pela transferência de fluídos da célula e do interstício, resultando em diminuição acentuada do hematócrito na fase aguda da reanimação ${ }^{2,5,49}$.

A comparação da solução de $\mathrm{NaCl}$ a $7,5 \%$ e de sua associação ao dextran 70 a $6 \%$ em cães submetidos a choque hemorrágico mostrou que somente no grupo em que se associou o dextran houve manutenção da expansão plasmática por mais de três horas da reanimação, com hematócrito diminuído ${ }^{8}$. Tal expansão plasmática teria origem pela redução do edema celular provocado pelo choque hemorrágico ${ }^{50,51}$. Também contribuiu para a expansão plasmática a remoção do fluido intracelular das hemácias e das células do endotélio vascular pelas soluções hipertônicas de cloreto de sódio ${ }^{52,53}$.

O efeito redutor de edema das células endoteliais foi particularmente importante nos capilares, onde o edema previamente instalado reduziu de modo significativo a luz do vaso, impondo restrição hemodinâmica significativa ao fluxo das hemácias. Este fato pode explicar a imediata retomada da função metabólica após a administração da solução hipertônica, em comparação com o mesmo efeito expansor de solução isotônica, na medida em que esta recompõe o volume circulante na mesma proporção, mas não remove de imediato o edema endotelial ${ }^{54-56}$.

O gradiente osmótico das soluções com $2.400 \mathrm{mOsm} \cdot \mathrm{L}^{-1} \mathrm{fez}$ com que houvesse a remoção de fluidos intracelulares das hemácias e das células endoteliais, primeiramente, e, posteriormente, do interstício e da célula tecidual. É provável que a adição da solução hiperoncótica de dextran tenha determinado, nesta pesquisa, que o efeito da expansão plasmática perdurasse no último momento do experimento, não permitindo a tendência de aumento do hematócrito, como se observou no $\mathrm{G} 2$.

$\mathrm{Na}$ dose de $4 \mathrm{~mL} \cdot \mathrm{kg}^{-1}$ e administração única, a SH adiciona $5,13 \mathrm{mEq} . \mathrm{L}^{-1}$ de sódio por quilo de peso, sendo a hipernatremia observada moderada e temporária ${ }^{57}$. Como o volume intravascular é de cerca de $40 \mathrm{~mL}^{\mathrm{kg}}{ }^{-1}$, essa carga de sódio administrada promoveria um aumento teórico de $128 \mathrm{mEq} . \mathrm{L}^{-1}$, elevando o sódio plasmático a $263-268 \mathrm{mEq} \cdot \mathrm{L}^{-1}$. No entanto, à medida que o soluto é administrado, o seu poder osmótico dilui o compartimento intravascular, evitando o aumento teoricamente esperado do sódio plasmático ${ }^{58}$.

Nesta pesquisa, o sódio plasmático apresentou elevação acentuada após a reanimação com as soluções de $\mathrm{NaCl}$ a $7,5 \%$ e com sua associação com dextran 70 a $6 \%$, chegando a valores de em torno de $153 \mathrm{mEq} \cdot \mathrm{L}^{-1}$. A elevação do sódio plasmático foi moderada e temporária, aumentando os valores em média em $12 \mathrm{mEq}$ L-1 $^{-1}$, não oferecendo riscos para os cães ${ }^{7,59}$. 
Concluiu-se que, neste modelo experimental em cães, a associação da solução hiperosmolar de cloreto de sódio a $7,5 \%$ à solução hiperoncótica de dextran 70 a 6\% (SHD) apresentou melhor desempenho hemodinâmico com relação à solução de $\mathrm{NaCl}$ a 7,5\% simples, sobretudo após 30 minutos do início da reanimação. A perfusão tecidual, observada pela saturação venosa mista e diferença arteriovenosa de oxigênio, foi melhor com a SHD do que com a SH. A expansão plasmática proporcionada pela elevada tonicidade das preparações ficou evidente no momento imediato à reanimação nos dois grupos, porém somente a associação ao dextran 70 a $6 \%$ fez com que este efeito perdurasse até 30 minutos após o início da administração das soluções.

\section{Immediate Hemodynamic and Metabolic Effects of $7.5 \%$ Sodium Chloride and its Association with 6\% Dextran 70 in Hemorragic Shock Resuscitation. An Ex- perimental Study in Dogs}

José Fernando Amaral Meletti, TSA, M.D.; José Reinaldo Cerqueira Braz, TSA, M.D.; Norma Sueli Pinheiro Módolo, TSA, M.D.

\section{INTRODUCTION}

Hypertonic sodium chloride has been used since the beginning of the XX Century as initial or adjuvant treatment of hemorrhagic shock. Its use was first described in the treatment of American soldiers in World War I ${ }^{1}$. In the 1960s, it was observed that sodium chloride solutions containing 1800 mOsm. $L^{-1}$ provided temporary recovery of the blood pressure in patients with hemorrhagic shock ${ }^{2}$. That result was attributed to volume expansion and pre-capillary vasodilation ${ }^{3,4}$.

Velasco et al. demonstrated that the administration of $7.5 \%$ sodium chloride solution (2400 mOsm. $\mathrm{L}^{-1}$ ), at a volume corresponding to $10 \%$ of the total blood loss, to dogs with hemorrhagic shock (blood loss of 40 to $50 \mathrm{~mL} . \mathrm{kg}^{-1}$ ) improved mean arterial blood pressure, cardiac output, and survival. Hemodynamic parameters did not improve in animals treated with normal saline and they all died ${ }^{5}$.

The effects of $7.5 \% \mathrm{NaCl}$ were temporary, disappearing one to two hours ${ }^{6,7}$ after the infusion. Associating 6\% dextran 70 to the hypertonic solution ${ }^{8}$ accelerated volume expansion and converted the temporary hemodynamic effects of the dextran solution into an effective increase in blood pressure and cardiac output ${ }^{9,10}$.

Other colloid solutions, besides dextran, were evaluated to determine whether their association was also beneficial. Hemodynamic responses were better for $7.5 \% \mathrm{NaCl}$ in $6 \%$ dextran 70 than for $7.5 \% \mathrm{NaCl}$ in $6 \%$ hetastarch ${ }^{11}$.
Comparative evaluation of $7.5 \% \mathrm{NaCl}$ solution and its association with $6 \%$ dextran 70 was studied in experimental models of hemorrhagic shock in pigs, in which some hemodynamic parameters were measured 5,15 , and 30 minutes after the treatment. It showed that the association with dextran was significantly superior to $7.5 \% \mathrm{NaCl}$ alone ${ }^{12}$.

Prospective, randomized studies evaluated the effects of $7.5 \% \mathrm{NaCl}$ alone or associated with $6 \%$ dextran 70 as the initial treatment of hemorrhagic shock in approximately 1,500 patients admitted to hospitals ${ }^{13-16}$. They demonstrated that the hypertonic solution associated with dextran was safe and free of toxic side effects. There were no renal, neurological, cardiopulmonary, or septic complications. They showed a fast and significant increase in blood pressure, longer survival, and absence of rebleeding ${ }^{17}$.

This study was designed to determine whether $7.5 \% \mathrm{NaCl}$ solution or its association with $6 \%$ dextran 70 presented different hemodynamic and metabolic effects in the immediate post-resuscitation period in the experimental model of controlled hemorrhagic shock in dogs, since the benefits of adding hyperoncotic solutions to hypertonic sodium chloride solutions are evident in the long-term control of hemorrhagic shock.

\section{METHODS}

After approval by the Ethics Committee on Animal Experiments of the Faculdade de Medicina da UNESP, Campus Botucatu, 16 adult, mongrel dogs, of both genders, weighing between 16 and $24 \mathrm{~kg}$ were studied. They were divided in two groups with 8 animals each, which underwent three experimental phases: in the preparation phase, the animals were treated with the same anesthetics and submitted to the same procedures; in the experimental resuscitation phase, the animals were divided according to the type of solution used for resuscitation:

G1: hypertonic solution: $7.5 \%$ sodium chloride solution (HS) $-4 \mathrm{~mL} . \mathrm{kg}^{-1}$.

G2: hypertonic solution with dextran: $7.5 \%$ sodium chloride solution $6 \%$ dextran 70 (HSD) -4 mL. $\mathrm{kg}^{-1}$.

After a 12-hour fasting period, animals were anesthetized with pentobarbital sodium $30 \mathrm{mg} \cdot \mathrm{kg}^{-1}$, with a maintenance anesthesia of $3.6 \mathrm{mg} \cdot \mathrm{kg}^{-1} \mathrm{~h}^{-1}$ (infusion pump model Anne, Abbot, USA), and placed on a Claude Bernard gutter and the following procedures were performed:

1. Tracheal intubation and controlled mechanical ventilation with a rebreather system using model 674 of the anesthesia device K. Takaoka model 2600. The settings included a tidal volume of $12 \mathrm{~mL} . \mathrm{kg}^{-1}$, respiratory rate of 16 irpm, and oxygen at 1 L. $\min ^{-1}$;

2. Dissection and catheterization of the femoral veins, left and right, for administration of anesthetic drugs (pentobarbital sodium and $0.08 \mathrm{mg} \cdot \mathrm{kg}^{-1}$ of pancuronium chloride) and to collect blood samples, respectively; 
3. Dissection and catheterization of the left femoral artery to monitor blood pressure, and right femoral artery to bleed the dog;

4. Dissection of the right external jugular artery and placement of a Swan-Ganz catheter;

5. Calibration of the Datex Engstron monitor, type D-VNC 1500-02 s/n 763177;

6. Verification of catheter placement;

7. Monitoring with continual electrocardiogram, $D_{\|}$lead derivation, pulse oxymeter on the animal's tongue, and tympanic thermometer on the left ear;

8. Laparotomy with a midline incision for splenectomy with strict homeostasis of the surgical wound;

9. Complementation of the dose of pancuronium $(0.02$ mg. $\mathrm{kg}^{-1}$ ) and stabilization of the animal for 10 minutes;

10. Performance of the first phase of the study (control phase): drawing blood samples: arterial, venous, and mixed; and determination of baseline hemodynamic parameters (heart rate, systolic arterial pressure, mean arterial pressure, pulmonary arterial pressure, central venous pressure, pulmonary capillary wedge pressure, cardiac output, cardiac index, systolic index, systemic vascular resistance index, pulmonary vascular resistance index, and left ventricle end-diastolic work load index);

11. Animals were bled two minutes after the control phase;

12. Bleeding $10 \%$ of the animal's calculated blood volume through the right femoral artery catheter and observation of the hemodynamic parameters for 5 minutes;

13. Bleeding of an additional $10 \%$ of the animal's calculated blood volume through the right femoral artery catheter and observation of the hemodynamic parameters for 5 minutes to determine whether the animal would need further bleeding;

14. Bleeding was discontinued when mean arterial pressure was maintained at approximately $40 \mathrm{mmHg}$ for a 5 -minute period;

15. Animals were stabilized for 30 minutes;

16. Drawing arterial, venous, and mixed blood samples and determination of the same hemodynamic parameters halfway the stabilization phase (M2);

17.4 mL. $\mathrm{kg}^{-1} \mathrm{HS}$ (G1) or HSD (G2) were administered for 3 minutes;

18. Collection of arterial, venous, and mixed blood samples and determination of hemodynamic parameters $2 \mathrm{mi}-$ nutes after the infusion of the hypertonic solution was finished (M3);

19. The animal was stabilized for 25 minutes;

20. Drawing arterial, venous, and mixed blood samples and the measurement of hemodynamic parameters $30 \mathrm{mi}-$ nutes after the beginning of the infusion of hypertonic solution (M4);

21. End of the experiment.

\section{Parameters Evaluated}

1. Hemodynamic: heart rate (HR - beats. $\left.\min ^{-1}\right)$, mean arterial pressure (MAP $-\mathrm{mmHg}$ ), pulmonary capillary wedge pressure (PCWP - mmHg), cardiac index $\left(\mathrm{Cl}-\mathrm{L} \cdot \mathrm{min}^{-1} \cdot \mathrm{m}^{-2}\right)$, systemic vascular resistance index (SVRI - dinas.seg. $\left.\mathrm{cm}^{-5} \cdot \mathrm{m}^{-2}\right)$.

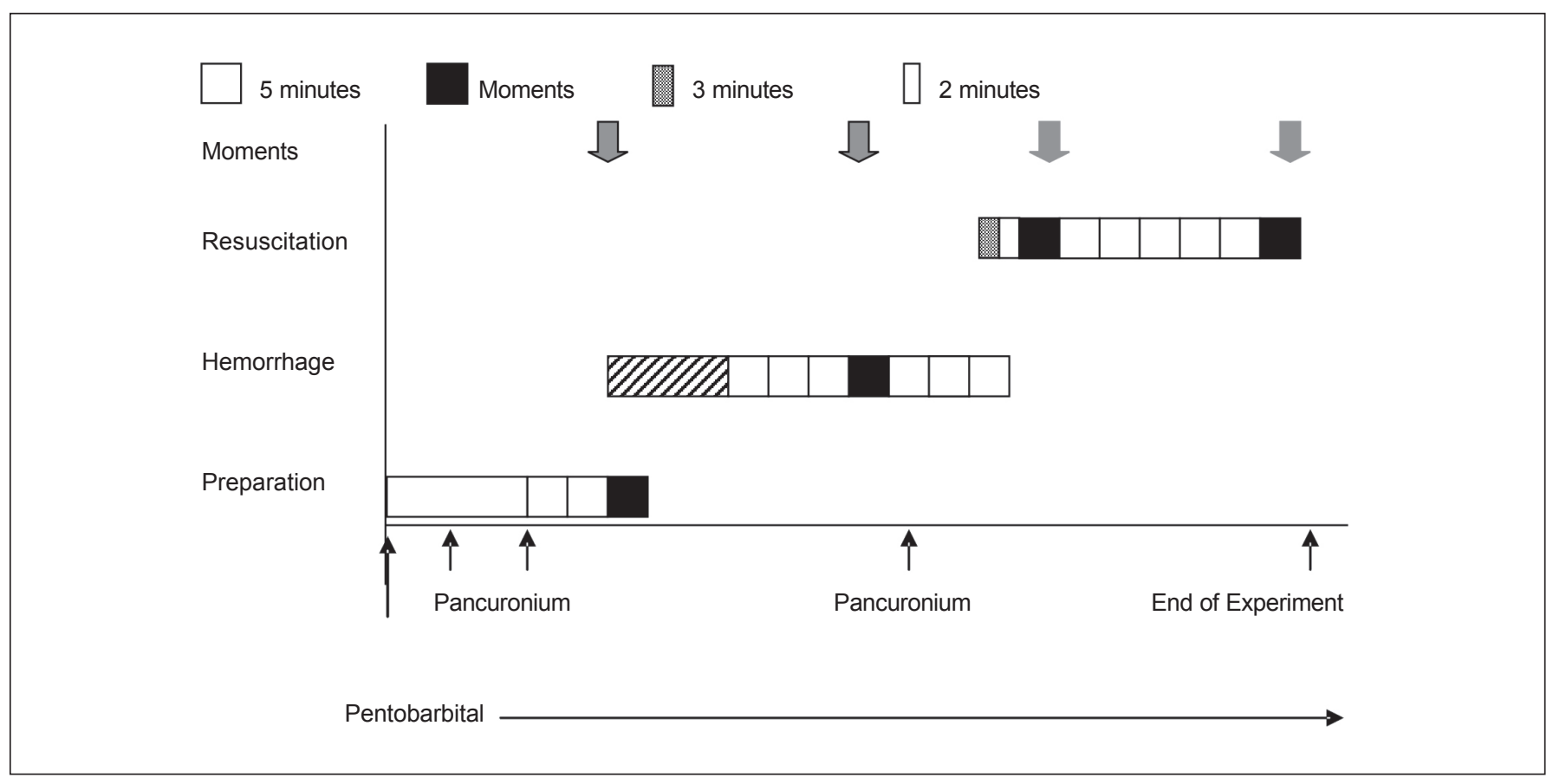

Figure 1 - Sequence of the Experiment 
2. Tissue oxygenation and metabolites: mixed venous saturation $\left(\mathrm{SvO}_{2}-\%\right)$, oxygen arteriovenous difference $\mathrm{C}_{(\text {av) }} \mathrm{O}_{2}$ $\mathrm{mL}$. $\left.100 \mathrm{~mL}^{-1}\right)$, oxygen delivery $\left(\mathrm{DO}_{2}-\mathrm{mL} \cdot \mathrm{min}^{-1}\right)$, oxygen consumption $\left(\mathrm{VO}_{2}-\mathrm{mL} \cdot \mathrm{min}^{-1}\right)$, plasma lactate (Lactate $\left.\mathrm{mEq} \cdot \mathrm{L}^{-1}\right)$.

3. Acid-base balance: $\mathrm{pH}$, partial pressure of carbon dioxide $\left(\mathrm{PaCO}_{2}-\mathrm{mmHg}\right)$.

4. Hematological: sodium plasma level $\left(\mathrm{Na}-\mathrm{mEq} \cdot \mathrm{L}^{-1}\right)$, hematocrit ( $\mathrm{Ht}-\%)$.

The data were measured in 4 moments: M1 - after a 10-minute stabilization period following the surgical phase, considered the baseline or control parameter; M2 - after the shock phase, 15 minutes after the beginning of the 30-minute stabilization period; M3 - 2 minutes after the infusion of the IV solution ended; M4 - 30 minutes after the beginning of the resuscitation with hypertonic solution.

\section{Statistical Analysis}

The statistical analysis of the weight, height, and volume of blood loss was done once and the comparison between both groups was done using the test $t$ for two independent samples, calculating $t$ and $\mathrm{p}^{18}$.

Profile Analysis was used to verify the variables through time $^{19}$. F and $p$ were calculated for each hypothesis. In every analysis, values were considered statistically significant for a $p<0.05$. When $0.05<p<0.10$ it was considered a significant tendency ( $p$ is the probability of wrongfully concluding for the significance).

\section{RESULTS}

Weight, length, body surface area, and volume of blood loss were similar in both groups (test t) (Table I).

Heart rate was lower at the end of the bleeding phase and in the beginning of the resuscitation phase in both groups, returning to baseline (control) values at the end of the experiment. Mean arterial pressure was increased in both groups after resuscitation (M3 and M4); however, these values did not return to baseline (M1). Pulmonary capillary wedge pressure was lower in G2 than in G1 at the second phase of the experiment, but values were the same in both groups during resuscitation. Even though both groups have similar profiles, $\mathrm{Cl}$ in $\mathrm{G} 2$ was slightly higher than in G1 during the fourth phase. Baseline SVRI values were increased in both groups, remained elevated after bleeding, but were significantly reduced at the beginning of the resuscitation; however, SVRI returned to baseline values at the end of the experiment (Table II).

The profile of $\mathrm{SvO}_{2}$ values was different between both groups and among the different phases. In phase four,

Table I - Weight, Lenght, Body Surface Area, and Volume of Bleeding

\begin{tabular}{|c|c|c|c|c|c|}
\hline Variables & $\mathrm{G} 1$ * & $\mathrm{G} 2$ * & $p$ value & $t$ value & Comments \\
\hline Lenght (cm) & $105.5 \pm 5.32$ & $101 \pm 8.19$ & $>0.1$ & 1.18 & $\mathrm{G} 1=\mathrm{G} 2$ \\
\hline Surface area $\left(\mathrm{m}^{2}\right)$ & $0.75 \pm 0.062$ & $0.73 \pm 0.086$ & $>0.1$ & 0.57 & $\mathrm{G} 1=\mathrm{G} 2$ \\
\hline Bleeding (mL) & $683.8 \pm 184.3$ & $680.6 \pm 157.5$ & $>0.5$ & 0.04 & $\mathrm{G} 1=\mathrm{G} 2$ \\
\hline
\end{tabular}

*Values expressed in mean $\pm \mathrm{SD}$

Table II - Hemodynamic Parameters

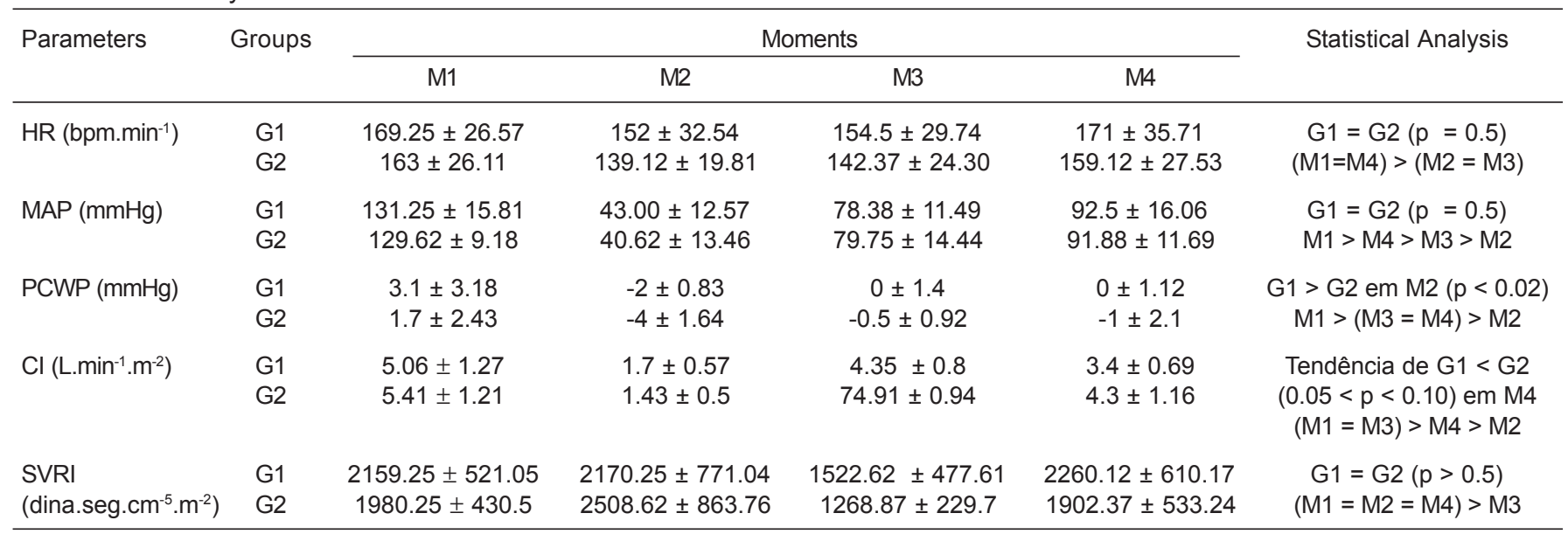

Heart Rate (HR), Mean Arterial Pressure (MAP), Pulmonary Capillary Wedge Pressure (PCWP), Cardiac Index (Cl), Systemic Vascular Resistance Index (SVRI). 


\section{IMMEDIATE HEMODYNAMIC AND METABOLIC EFFECTS OF 7.5\% SODIUM CHLORIDE AND ITS ASSOCIATION WITH 6\% DEXTRAN 70 IN HEMORRAGIC SHOCK RESUSCITATION. AN EXPERIMENTAL STUDY IN DOGS}

those values were significantly higher in $\mathrm{G} 2$ than in $\mathrm{G} 1$ Regarding the oxygen content difference, values in $\mathrm{G} 1$ were significantly higher during resuscitation. Baseline $\mathrm{DO}_{2}$ values were elevated in both groups. They decreased drastically during the shock, but increased after crystalloid expansion without reaching baseline values. Oxygen consumption values were higher in G1 than in G2. Plasma lactate values increased gradually until the beginning of the resuscitation; at the end of the experiment, it reduced considerably to values similar to those found during the hypovolemic shock (Table III).
As for the acid-base balance, $\mathrm{pH}$ values did not change with the hemorrhagic shock, decreasing after crystalloid expansion. After resuscitation, the partial pressure of carbon dioxide increased considerably in both groups (Table IV).

Both groups showed equal and pronounced elevation of sodium plasma values after volume resuscitation. On the other hand, hematocrit values reduced significantly after bleeding and immediately before resuscitation, stabilizing after the administration of IV solution in both groups (Table V).

Table III - Tissue Oxygenation and Metabolic Parameters

\begin{tabular}{|c|c|c|c|c|c|c|}
\hline \multirow[t]{2}{*}{ Parameters } & \multirow[t]{2}{*}{ Groups } & \multicolumn{4}{|c|}{ Moments } & \multirow[t]{2}{*}{ Statistical Analysis } \\
\hline & & M1 & M2 & M3 & M4 & \\
\hline $\mathrm{SvO}_{2}(\%)$ & $\begin{array}{l}\text { G1 } \\
\text { G2 }\end{array}$ & $\begin{array}{l}0.9 \pm 0.04 \\
0.93 \pm 0.02\end{array}$ & $\begin{array}{c}0.58 \pm 0.1 \\
0.55 \pm 0.16\end{array}$ & $\begin{array}{l}0.79 \pm 0.04 \\
0.84 \pm 0.07\end{array}$ & $\begin{array}{l}0.73 \pm 0.12 \\
0.84 \pm 0.07\end{array}$ & $\begin{array}{c}\mathrm{G} 1<\mathrm{G} 2 \text { em M4 }(p<0.05) \\
\mathrm{M} 1>(\mathrm{M} 3=\mathrm{M} 4) . \mathrm{M} 2\end{array}$ \\
\hline $\begin{array}{l}\mathrm{C}_{(\mathrm{a}-\mathrm{v})} \mathrm{O}_{2} \\
\left(\mathrm{~mL} .100 \mathrm{~m}^{-1}\right)\end{array}$ & $\begin{array}{l}\text { G1 } \\
\text { G2 }\end{array}$ & $\begin{array}{l}2.74 \pm 1.15 \\
2.08 \pm 0.28\end{array}$ & $\begin{array}{c}8.28 \pm 2.07 \\
8.49 \pm 1.6\end{array}$ & $\begin{array}{l}2.84 \pm 0.54 \\
1.81 \pm 0.71\end{array}$ & $\begin{array}{c}4.2 \pm 2.05 \\
3.16 \pm 1.26\end{array}$ & $\begin{array}{c}\mathrm{G} 1>\mathrm{G} 2 \text { em M3 e } \\
\mathrm{M} 4(\mathrm{p}<0.01)(\mathrm{M} 1=\mathrm{M} 3) \\
<\mathrm{M} 4<\mathrm{M} 2\end{array}$ \\
\hline $\mathrm{VO}_{2}\left(\mathrm{~mL} \cdot \mathrm{m}^{-1}\right)$ & $\begin{array}{l}\mathrm{G} 1 \\
\mathrm{G} 2\end{array}$ & $\begin{array}{l}96.27 \pm 12.21 \\
80.48 \pm 11.38\end{array}$ & $\begin{array}{c}103.16 \pm 41.09 \\
91.10 \pm 44.11\end{array}$ & $\begin{array}{l}90.65 \pm 14.94 \\
63.79 \pm 29.85\end{array}$ & $\begin{array}{c}120.39 \pm 37.83 \\
97.73 \pm 47.66\end{array}$ & $\begin{array}{c}\mathrm{G} 1 \geq \mathrm{G} 2 \text { em M1 e M3 } \\
0.05<\mathrm{p}<0.1 \mathrm{M} 3< \\
(\mathrm{M} 1=\mathrm{M} 2)<\mathrm{M} 4\end{array}$ \\
\hline $\begin{array}{l}\text { Lactate } \\
\left(\mathrm{mEq} \cdot \mathrm{L}^{-1}\right)\end{array}$ & $\begin{array}{l}\mathrm{G} 1 \\
\mathrm{G} 2\end{array}$ & $\begin{array}{l}1.24 \pm 0.85 \\
0.93 \pm 0.60\end{array}$ & $\begin{array}{l}1.93 \pm 1.0 \\
1.94 \pm 1.15\end{array}$ & $\begin{array}{l}2.62 \pm 0.99 \\
2.23 \pm 0.91\end{array}$ & $\begin{array}{l}1.93 \pm 0.66 \\
1.48 \pm 0.44\end{array}$ & $\begin{array}{c}\mathrm{G} 1=\mathrm{G} 2 \mathrm{M} 1<(\mathrm{M} 2=\mathrm{M} 4) \\
<\mathrm{M} 3\end{array}$ \\
\hline
\end{tabular}

Venous Mixed Saturation $\left(\mathrm{SvO}_{2}\right)$, Oxygen Arteriovenous Difference $\left(\mathrm{C}_{(a v)} \mathrm{O}_{2}\right)$, Oxygen Delivery $\left(\mathrm{DO}_{2}\right)$, Oxigen Consumption $\left(\mathrm{VO}_{2}\right)$ and Plasma Lactate Level (lactate)

Table IV - Parameters of Acid-Base Balance

\begin{tabular}{lcccccc}
\hline Parameters & Groups & \multicolumn{2}{c}{ Moments } & \multicolumn{2}{c}{ Statistical Analysis } \\
\cline { 3 - 6 } & & M1 & M2 & M3 & M4 \\
\hline $\mathrm{pH}$ & $\mathrm{G} 1$ & $7.28 \pm 0.06$ & $7.24 \pm 0.07$ & $7.13 \pm 0.11$ & $7.17 \pm 0.08$ & $\mathrm{G} 1=\mathrm{G} 2$ \\
& $\mathrm{G} 2$ & $7.32 \pm 0.07$ & $7.22 \pm 0.1$ & $7.10 \pm 0.11$ & $7.5 \pm 0.09$ & $(\mathrm{M} 1=\mathrm{M} 2)>(\mathrm{M} 3=\mathrm{M} 4)$ \\
$\mathrm{PaCO}_{2}$ & $\mathrm{G} 1$ & $49.75 \pm 11.69$ & $45.12 \pm 10.26$ & $66.66 \pm 15.64$ & $64.83 \pm 16.83$ & $\mathrm{G} 1=\mathrm{G} 2$ \\
& $\mathrm{G} 2$ & $49.36 \pm 13.86$ & $50.55 \pm 11.43$ & $71.48 \pm 12.11$ & $61.87 \pm 8.4$ & $(\mathrm{M} 1=\mathrm{M} 2)<(\mathrm{M} 3=\mathrm{M} 4)$ \\
\hline
\end{tabular}

Arterial Blood pH $(\mathrm{pH})$, Partial Pressure of Carbon Dioxide $\left(\mathrm{PaCO}_{2}\right)$

Table V - Hematologic Parameters

\begin{tabular}{|c|c|c|c|c|c|c|}
\hline Parameters & Groups & \multicolumn{4}{|c|}{ Moments } & Statistical Analysis \\
\hline Plasma sodium & $\mathrm{G} 1$ & $145.5 \pm 5.04$ & $140.5 \pm 8.66$ & $153.5 \pm 8.89$ & $148.5 \pm 4.75$ & $\mathrm{G} 1>\mathrm{G} 2 \mathrm{em} \mathrm{M} 1$ \\
\hline
\end{tabular}




\section{DISCUSSION}

The anesthetic technique used is recommended by most authors for this experimental model; it allows the establishment of an adequate anesthetic plane in a few minutes ${ }^{5,20-23}$. Pentobarbital sodium was infused continuously $\left(3.6 \mathrm{mg} . \mathrm{kg}^{-1}\right.$. $\mathrm{h}^{-1}$ ) to maintain a stable plasma concentration of the drug to keep the dog anesthetized during the whole experiment ${ }^{24-26}$. The experimental model of hemorrhagic shock in animals used frequently is the Wiggers, 1942 model ${ }^{27}$. Shock is induced by bleeding the animal and maintaining the mean arterial pressure between 35 and $40 \mathrm{mmHg}$. Several researchers have modified this model to suit the objectives of their work ${ }^{28-30}$.

It was determined that the hypertonic solution would be administered over a 3-minute period, similar to other works ${ }^{8,9,13,31}$, and this time was aimed at not causing histological lesions in the vascular wall ${ }^{31}$. The volume infused to each group was $4 \mathrm{~mL} \cdot \mathrm{kg}^{-1}$. This is the volume proposed by most studies, experimental or in human beings $5,7,8,11,32$.

We observed that the heart rate decreased significantly in the hemorrhagic shock phase. This can be explained by the Baindridge reflex in which a decrease in venous return leads to bradycardia, since the sensibility of the venous baroreceptors is greater than the arterial baroreceptors ${ }^{33,34}$. Therefore, in this situation, the significative reduction in venous return may have caused a reduction in heart rate.

At the time of resuscitation with both solutions used in this study, there were no changes in HR, which was also observed in other studies using rapid infusion of hypertonic solutions ${ }^{12,35}$. Thirty minutes after the infusion of the IV solution started, plasma osmolarity increased due to an increase in sodium concentration, leading to improved cardiac performance and HR when compared to control levels ${ }^{23,36}$.

The simple hypertonic solution improves hemodynamic parameters temporarily, unless a colloid solution is added to the hyperosmolar solution. While the hypertonicity of the solution is responsible for removing fluid from the intracellular space, the hyperoncotic colloid solution maintains this fluid in the intravascular space. The physiological effects of small volumes of hypertonic solutions are associated with an increase in mean arterial pressure, cardiac output, and plasma expansion. These solutions also increase oxygen consumption; dilate the pre-capillary sphincters; increase cardiac contractility, diuresis, and natriuresis; restore membrane potential and decrease the volume needed to restore blood volume in the long-term control of shock ${ }^{11}$. In this study, both solutions had similar hemodynamic performances in the second minute after the administration of the solution finished, earlier than other reports in the literature. However, from that point until 30 minutes after the beginning of resuscitation, the superiority of the hypertonic solution in dextran is unquestionable, especially when the cardiac index in both groups is analyzed.
The effects of the $7.5 \%$ hypertonic solution on blood pressure, cardiac output, and systolic index are almost instantaneous, lasting 45 minutes for $7.5 \% \mathrm{NaCl}$ and from 2 to 4 hours when it is combined with $6 \%$ dextran 70 . The increase in cardiac contractility does not depend on preload ${ }^{17}$.

Vasodilation is most likely secondary to an effect of the osmolarity on the arteriolar wall or to a vasodilation reflex triggered by pulmonary receptors that can detect an increase in plasma osmolarity. Characteristically, vasodilation triggered by hypertonic solutions decreases systemic vascular resistance and pulmonary vascular resistance directly, independently of the local innervation, and is observed in most vascular beds ${ }^{17}$.

When hemodynamic parameters were analyzed, we noticed that hyperosmolar solutions at $7.5 \%$ produced immediate and peculiar cardiovascular effects. Basically, there was an important pre-capillary vasodilation, with decreased systemic vascular resistance, improved myocardial contractility, increased mean arterial pressure and pulmonary capillary wedge pressure, and increased preload indexes.

Adding the hyperoncotic solution, $6 \%$ dextran 70 , to $7.5 \%$ $\mathrm{NaCl}$ did not produce any hemodynamic differences during the early resuscitation phase (M3). Analyzing the results 30 minutes after the resuscitation began, HSD had better vascular performance, especially in the cardiac index.

In normal physiological conditions, oxygen consumption is determined by the tissue metabolic needs, independently of oxygen transport. During periods of increased metabolism or decreased tissue perfusion, oxygen extraction, which is normally $25 \%$, increases to $75 \%-80 \%$. In these conditions, there is a drastic reduction in oxygen content in the central compartment ${ }^{37}$.

In this study, there was a $38 \%$ reduction in the mixed venous saturation with a $46 \%$ reduction in blood volume during the hemorrhagic shock phase. This result was similar to the results reported in the literature ${ }^{28,38}$.

Mixed venous saturation showed the same results in both groups in the resuscitation phase. However, at the end of the experiment, they remained unchanged in the HSD group, while they were significantly reduced in the HS group. These results demonstrate that $7.5 \% \mathrm{NaCl}$ in dextran 70 was superior to the hypertonic solution alone regarding mixed venous saturation in the first 30 minutes of resuscitation. Mixed venous saturation demonstrated that blood flow in the microcirculation was even lower in the HS group, leading to an increased oxygen extraction, than in the HSD group.

Oxygen delivery $\left(\mathrm{DO}_{2}\right)$ is the result of the arterial oxygen content $\left(\mathrm{CaO}_{2}\right)$ multiplied by the cardiac output $(\mathrm{CO})$. Conversely, the reduction in arterial oxygen content depends on hemoglobin oxygen concentration and saturation. Therefore, a reduction in $\mathrm{DO}_{2}$ can be corrected with a blood transfusion or increasing cardiac output. Cardiac output tends to be increased when oxygen consumption is increased, such as in trauma ${ }^{39}$. Oxygen consumption $\left(\mathrm{VO}_{2}\right)$ is the result of oxygen venous content multiplied by cardiac output. Reduced or inadequate 
oxygen consumption is the common denominator in every shock syndrome. Oxygen consumption also seems to be related with survival in patients with hemorrhagic shock ${ }^{40}$. The oxygen content arteriovenous difference index $\left(\mathrm{C}_{(\mathrm{av})} \mathrm{O}_{2}\right)$ represents the relationship between oxygen consumption and cardiac output. For this reason, it represents intrinsically an index of hemodynamic function adequacy; therefore, its elevation usually represents hypodinamic cardiovascular states secondary to cardiac dysfunction or hypovolemia ${ }^{41}$. With a blood loss of approximately $34 \mathrm{~mL} . \mathrm{kg}^{-1}$, we observed a reduction in oxygen delivery and increase in arteriovenous oxygen difference in both groups, but oxygen consumption remained unchanged. Oxygen consumption should be considered a prognostic index in hemorrhagic shock. When reduced, it indicates irreversible shock ${ }^{3}$.

Hannon et al. ${ }^{42}$ evaluated the relationship between oxygen transport and requirements during a hemorrhage of approximately $37 \mathrm{~mL} . \mathrm{kg}^{-1}$ in one hour and during resuscitation with $4 \mathrm{~mL} . \mathrm{kg}^{-1}$ of $7.5 \% \mathrm{NaCl}$ in $6 \%$ dextran 70 in the experimental shock model in pigs. During the shock phase, there was a slight increase in $\mathrm{VO}_{2}$, lactate plasma values were significantly increased, $\mathrm{DO}_{2}$ values were significantly decreased, and oxygen requirements doubled. The difference between oxygen delivery and requirements was reduced by volume expansion with hypertonic solution, primarily by the suppression of the metabolic requirements and, to a lesser extent, by an increase in $\mathrm{DO}_{2}$.

Hemodynamic and metabolic performance of HS and HSD solutions at 5,15 , and 30 minutes after resuscitation of pigs with hemorrhagic shock demonstrated that oxygen transport values were significantly higher in the dextran group throughout the experiment.

Clinically, increased lactate values presented a good correlation with oxygen deficiency and anaerobic metabolism. In humans, lactate values higher than $2 \mathrm{mEq} \cdot \mathrm{L}^{-1}$ are associated with increased mortality, and values higher than 3 and 4 $\mathrm{mEq} . \mathrm{L}^{-1}$ indicate profound hypoperfusion.

In aerobic conditions, carbohydrate metabolism follows the Krebs cycle; therefore, the sequential oxidation of a molecule of carbon produces 38 ATP molecules. When oxygen supply is reduced, the usual sequence is interrupted. Pyruvate is converted in lactate and the energy yield is limited to the production of just 2 ATP molecules. Lactate produced in anaerobic conditions accumulates in the body because it is used in a limited number of depurative processes that occur, basically, in the hepatocytes ${ }^{37}$.

Strecker et al. ${ }^{43}$ studied the efficacy of $6 \%$ dextran or $6 \%$ hetastarch associated with $7.5 \%$ hypertonic solutions in the resuscitation of hemorrhagic shock in rabbits. In both groups, the oxygen content difference returned to pre-shock values 2 minutes after resuscitation, similar to what happened in our study. But in his study, they were significantly increased in the hetastarch group 15 and 60 minutes after resuscitation. Serum lactate values decreased immediately in both groups, but this reduction was less prominent in the dextran group. Lactate and BE values were significantly reduced, but this reduction was greater in the dextran group than in the hetastarch group 60 minutes after resuscitation. In Strecker's study, a reduction in circulating volume caused a reduction in cardiovascular function that led to a significant decrease in $\mathrm{TEO}_{2}$ during the hemorrhagic shock phase. After resuscitation, $\mathrm{CO}$ and $\mathrm{DO}_{2}$ were higher in the group that received hypertonic saline-dextran (HSD). Consequently, the oxygen content difference was lower in the HSD group than in the hetastarch group. The authors explained that the greater elevation of lactate values in the HSD group was secondary to an increase in tissue perfusion in areas with high lactate content caused by the shock.

In this study, $\mathrm{C}_{(a v)} \mathrm{O}_{2}$ values were higher in the hypertonic solution group on both resuscitation times. The $\mathrm{TEO}_{2}$ values showed a statistically significant difference; they were higher in G1 30 minutes after IV infusion started. It indicated that the decrease in systemic flow was greater in $\mathrm{G} 1$ than in $\mathrm{G} 2$, being responsible for the greater oxygen extraction by the cells and the smaller mixed venous saturation in $\mathrm{G} 1$ seen at the end of the experiment.

In this study, there were significant differences in lactate values in the study groups, which were reduced only at the end of the experiment.

Chiara et al. ${ }^{44}$ also compared $7.5 \% \mathrm{NaCl}$ and its combination with $6 \%$ dextran 70 in an experimental model of hemorrhagic shock in pigs. They concluded that there were no statistically significant differences in $\mathrm{DO}_{2}, \mathrm{VO}_{2}$, and $\mathrm{TEO}_{2}$ between both solutions in the beginning of the resuscitation. These indexes increased significantly only one hour after the administration of the solutions in the HSD group when compared to the HS group.

There was an decrease in $\mathrm{pH}$ and an increase in the partial pressure of carbon dioxide after the administration of the hypertonic solution of sodium chloride $\left(1800 \mathrm{mOsm} \cdot \mathrm{L}^{-1}\right)^{2}$. The same happened after the administration of $7.5 \% \mathrm{NaCl}^{5}$. Arterial $\mathrm{pH}$ changed from 7.34 to 7.26 after removal of around $44 \%$ of the estimated blood volume for each dog ${ }^{45}$. In our study, $\mathrm{pH}$ was reduced from about 7.30 to 7.23 in both groups, and there were no statistically significant differences in the variation of $\mathrm{pH}$ after the removal of approximately $46 \%$ of the estimated blood volume for each dog.

In our experiment, after volume expansion, both groups presented similar behavior. The increase in $\mathrm{pH}$ and partial pressure of carbon dioxide was significant and sustained, both in M3 and M4, similar to what was seen in other studies ${ }^{42,43}$. The administration of hypertonic solution alone or in dextran increased the $\mathrm{PaCO}_{2}$ and reduced arterial $\mathrm{pH}$ significantly shortly after resuscitation of hemorrhagic shock. This was due to the brief acidemia caused, mainly, by the hyperchloremia, hypokalemia, and metabolic acidosis without anion gap, secondary to respiratory acidosis caused by elevated $\mathrm{PaCO}_{2}{ }^{46,47}$.

In our study, the worsening of the acidosis in the initial stages of resuscitation was probably due to a possible hyperchlo- 
remia caused by the administration of hypertonic solution, without enough time for its recovery. The sudden increase in partial pressure of carbon dioxide indicated improvement in tissue perfusion, but also contributed to maintaining the acidemia recorded during resuscitation.

Substituting chloride for acetate in the hypertonic solution, in order to produce isochloremic resuscitation, was not effective. Plasma chloride values were not elevated and blood acidosis was quickly corrected, but its administration led to unsatisfactory cardiovascular and metabolic performances when compared with traditional solutions ${ }^{48}$.

There was a reduction in hematocrit immediately after resuscitation. This was explained by the acute expansion of the intravascular compartment caused by the administration of $7.5 \% \mathrm{NaCl}$ and by the transport of fluids from the intracellular and interstitial compartments, resulting in a dramatic reduction in hematocrit immediately after resuscitation 2,5,49. Comparing $7.5 \% \mathrm{NaCl}$ with its association with $6 \%$ dextran 70 in dogs submitted to hemorrhagic shock showed that volume expansion was maintained over three hours in the group that received the hypertonic saline-dextran solution, with consequent decrease in hematocrit ${ }^{52,53}$. This plasma expansion would be secondary to the reduction of the cellular edema caused by hemorrhagic shock ${ }^{50,51}$. The hypertonic solution of sodium chloride also contributed with the removal of intracellular fluid from red blood cells and endothelial cells ${ }^{52,53}$. Reduction of the edema of the endothelial cells was particularly important in the capillaries, where previously settled edema reduced significantly vessel lumen, leading to an important reduction in red blood cell flow. This may explain the immediate recovery in metabolic function after the administration of hypertonic solution when compared with the same volume-expanding effect of the isotonic solution, since it recomposes the volume equally, but does not decrease endothelial edema immediately ${ }^{54-56}$.

The osmotic gradient of solutions with 2400 mOsm. $\mathrm{L}^{-1}$ removed intracellular fluid, initially from the red blood cells and from endothelial cells, followed by the interstitial compartment and tissue cells. It is possible that the addition of the hyperoncotic solution of dextran was responsible for the sustained plasma expansion at the end of the experiment, seen in G2, not allowing the hematocrit to rise.

The single administration of $4 \mathrm{~mL} \cdot \mathrm{kg}^{-1}$ of $\mathrm{HS}$ adds 5.13 mEq.L-1 of sodium per kilogram of weight, leading to a moderate and temporary hypernatremia ${ }^{57}$. Since intravascular volume is approximately $40 \mathrm{~mL} \cdot \mathrm{kg}^{-1}$, this sodium load would promote a theoretical increase of $128 \mathrm{mEq} . \mathrm{L}^{-1}$, elevating sodium plasma values to $263-268 \mathrm{mEq} . \mathrm{L}^{-1}$. However, while the solute is being administered, its osmotic force dilutes the intravascular compartment, avoiding the expected increase in plasma sodium concentration ${ }^{58}$.

In this study, sodium plasma values increased dramatically after resuscitation with $7.5 \% \mathrm{NaCl}$ and its association with $6 \%$ dextran 70 , reaching up to $153 \mathrm{mEq} \cdot \mathrm{L}^{-1}$. The elevation of plasma sodium values was moderate and temporary, in- creasing values an mean of $12 \mathrm{mEq} \cdot \mathrm{L}^{-1}$, which was not harmful for the dogs ${ }^{7,59}$.

We conclude that, in this experimental model in dogs, the association of a hypertonic solution, $7.5 \%$ sodium chloride, with a hyperosmolar solution, $6 \%$ dextran 70 (HSD), had a better hemodynamic performance than $7.5 \% \mathrm{NaCl}$ alone, especially 30 minutes after the beginning of the resuscitation. Tissue perfusion, reflected by the mixed venous saturation and arterial-venous oxygen difference, was better with HSD than with HS. Plasma expansion, secondary to the increased tonicity of the solutions, was evident immediately after resuscitation in both groups, but only the association with $6 \%$ dextran 70 was capable of maintaining this effect for up to 30 minutes after the beginning of the IV administration.

\section{REFERÊNCIAS - REFERENCES}

01. Penfield WG - The treatment of severe and progressive hemorrhage by intravenous injections. Am J Physiol, 1919;48:121128.

02. Baue AE, Tragus ET, Parkins WM - Effects of sodium chloride and bicarbonate in shock with metabolic acidosis. Am J Physiol, 1967;212:54-60.

03. Gazitua S, Scott JB, Chou CC et al - Effect of osmolarity on canine renal vascular resistance. Am J Physiol, 1969;217:1216-1223.

04. Gazitua S, Scott JB, Swindall B et al - Resistance response to local changes in plasma osmolality in three vascular beds. Am J Physiol, 1971;220:384-391.

05. Velasco IT, Pontieri V, Rocha e Silva M Jr et al - Hyperosmotic $\mathrm{NaCl}$ and severe hemorrhagic shock. Am J Physiol, 1980; 239: H664- H673.

06. Rocha e Silva M, Negraes GA, Soares AM et al - Hypertonic resuscitation from severe hemorrhagic shock: patterns of regional circulation. Circ Shock, 1986;19:165-175.

07. Rocha e Silva M, Velasco IT, Nogueira da Silva RI et al - Hyperosmotic sodium salts reverse severe hemorrhagic shock: other solutes do not. Am J Physiol, 1987;253:H751- H762.

08. Smith GJ, Kramer GC, Perron P et al - A comparison of several hypertonic solutions for resuscitation of bled sheep. J Surg Res, 1985;39:517-528.

09. Kramer GC, Perron PR, Lindsey DC et al - Small-volume resuscitation with hypertonic saline dextran solution. Surgery, 1986;100:239-247.

10. Velasco IT, Rocha e Silva M, Oliveira MA et al - Hypertonic and hyperoncotic resuscitation from severe hemorrhagic shock in dogs: a comparative study. Crit Care Med, 1989;17:261-264.

11. Kramer GC, Walsh JC, Perron PR et al - Comparison of hypertonic saline/dextran versus hypertonic saline/hetastarch for resuscitation of hypovolemia. Braz J Med Biol Res, 1989;22:279-282.

12. Wade C, Hannon J, Bossone $C$ et al - Superiority of hypertonic saline/dextran over hypertonic saline during the first $30 \mathrm{~min}$ of resuscitation following hemorrhagic hypotension in conscious swine. Resuscitation, 1990;20:49-56.

13. Holcroft JW, Vassar MJ, Turner $\mathrm{J}$ et al $-3 \% \mathrm{NaCl}$ and $7.5 \% \mathrm{Na} /$ $\mathrm{Cl} /$ dextran 70 in the resuscitation of severely injured patients. Ann Surg, 1987;206:276-288.

14. Mattox KL, Maningas PA, Moore EE et al - Prehospital hypertonic saline/dextran infusion for post-traumatic hypotension. The USA Multicenter Trial. Ann Surg, 1991;213:482-491.

15. Vassar MJ, Holcroft JW - Use of hypertonic-hyperosncotic fluids for resuscitation of trauma patients. J Intensive Care Med, 1992; 7:189-198. 


\section{IMMEDIATE HEMODYNAMIC AND METABOLIC EFFECTS OF 7.5\% SODIUM CHLORIDE AND ITS ASSOCIATION WITH 6\% DEXTRAN 70 IN HEMORRAGIC SHOCK RESUSCITATION. AN EXPERIMENTAL STUDY IN DOGS}

16. Younes RN, Ching CT, Goldenberg DC et al - Hypertonic salinedextran in the treatment of hemorrhagic shock: clinical trial in the emergency room. Proceedings of the $5^{\text {th }}$ International Conference on Hypertonic Resuscitation, 1992.

17. Rocha e Silva M - Hypertonic saline resuscitation. Medicina (B Aires), 1998;58:393-402.

18. Curi PR - Metodologia e Análise da Pesquisa em Ciências Biológicas. $2^{\mathrm{a}} \mathrm{Ed}$, Botucatu: Tipomic, 1998.

19. Morrison DF - Multivariate Statistical Methods. $3^{\text {a }}$ Ed, New York: Mc Graw Hill; 1967.

20. Gilmore JP - Pentobarbital sodium anesthesia in the dog. Am J Physiol, 1965;209:404-408.

21. Olmsted F, Page IH - Hemodynamic changes in dogs caused by sodium pentobarbital anesthesia. Am J Physiol, 1966;210:817820.

22. Priano LL, Traber DL, Wilson RD - Barbiturate anesthesia: an abnormal physiologic stimulation. J Pharmacol Exp Ther, 1969;165:126-135

23. Ogino R, Suzuki K, Kohno M et al - Effects of hypertonic saline and dextran 70 on cardiac contractility after hemorrhagic shock. J Trauma, 1998;44:59-69.

24. Hotvedt R, Platou ES, Koppang ER et al - Pentobarbital plasma concentration and cardiac eletrophysiology during prolonged pentobarbital infusion anesthesia in the dog. Acta Anaesthesiol Scand, 1982;26:638-642.

25. Welte M, Goresch T, Frey L et al - Hypertonic saline dextran does not increase cardiac contractile function during small volume resuscitation from hemorrhagic shock in anesthetized pigs. Anesth Analg, 1995;80:1099-1107.

26. Sato JK - Efeitos hemodinâmicos da isradipina em cães submetidos a pinçamento aórtico infra renal. [dissertação]. Botucatu: Faculdade de Medicina, Universidade Estadual Paulista; 1997.

27. Wiggers CJ, Werle JN - Exploration of a method for standarding hemorragic. Proc Soc Biol Med, 1942;49:604-606.

28. Dillon AR, Hankes GH, Nachreiner RF et al - Experimental hemorrhage in splenectomized and nosplenectomized dogs. Am J Vet Res, 1980;41:707-711.

29. Scalea TM, Holman M, Fuortes $M$ et al - Central venous blood oxygen saturation: an early, accurate measurement of volume during hemorrhage. J Trauma, 1988;28:725-732.

30. Pianim NA, Liu SY, Dubecz $S$ et al - Tissue oxygenation in hypovolemic shock. J Surg Res, 1993;55:338-343.

31. Hands R, Holcroft J, Perron P et al - Comparison of peripheral and central infusions of $7.5 \% \mathrm{NaCl} / 6 \%$ dextran 70 . Surgery, 1988;103:684-689.

32. Onarheim $H$, Missavage AE, Kramer GC et al - Effectiveness of hypertonic saline-dextran 70 for initial fluid resuscitation of major burns. J Trauma, 1990;30:597-603.

33. Epstein SE, Stampfer M, Beiser GD - Role of the capacitance and resistance vessels in vasovagal syncope. Circulation, 1968; 37:524-533.

34. Greene NM - Reports of scientific meetings. Anesthesiology, 1971;34:104-105.

35. De Barros LF, Baena RC, Velasco IT et al - Efeitos hemodinâmicos imediatos da infusão rápida de solução hipertônica de cloreto de sódio-dextran-70 para tratamento de choque hemorrágico em cães. Arq Bras Cardiol, 1993;61:317-320.

36. Mouren S, Delayance S, Mion G et al - Mechanisms of increased myocardial contractility with hypertonic saline solutions in isolated blood-perfused rabbit hearts. Anesth Analg, 1995;81:777-782.

37. Guyton AC - Tratado de Fisiologia Médica. $4^{a}$ Ed, Rio de Janeiro: Guanabara Koogan; 1992.

38. Kim SI, Desai JM, Shoemaker WC - Sequence of cardiorespiratory alterations after gradual prolonged hemorrhage in conscious dogs. Am J Physiol, 1969;216:1044-1050.
39. Barash PG, Lawrence LP - Clinical Anaesthesia. $5^{\text {th }}$ Ed, Philadelphia: Lippincott; 1993.

40. Shoemaker WC - Relation of oxygen transport patterns to the pathophysiology and therapy of shock states. Intensive Care Med, 1987:13:230-243.

41. Terzi RGG, Araújo S - Monitorização Hemodinâmica e Suporte Cardiocirculatório do Paciente Crítico. São Paulo: Atheneu; 1996.

42. Hannon JP, Wade CE, Bossone CA et al - Blood gas and acidbase status of conscious pigs subjected to fixed-volume hemorrhage and resuscitated with hypertonic saline dextran. Circ Shock, 1990;32:19-29.

43. Strecker U, Dick W, Madjidi A et al - The effect of type of colloid in the efficacy of hypertonic saline colloid mixture in hemorrhagic shock: dextran versus hydroxyethyl starch. Resuscitation, 1993;25:41-57.

44. Chiara O, Segala M, Volpi D et al - Hypertonic saline solutions in resuscitation in hemorrhagic shock. An experimental study. Minerva Chir, 1997;52:753-762.

45. Schwartz S, Frantz RA, Shoemaker WC - Sequential hemodynamic and oxygen transport responses in hypovolemia, anemia and hypoxia. Am J Physiol, 1981;241:H864-H871.

46. Dubick MA, Davis JM, Myers T et al - Dose response effects of hypertonic saline and dextran on cardiovascular responses and plasma volume expansion in sheep. Shock, 1995;3:137-144.

47. Moon PF, Kramer GC - Hypertonic saline-dextran resuscitation from hemorrhagic shock induces transient mixed acidosis. Crit Care Med, 1995;23:323-331.

48. Kramer GC, Elgjo GI, De Figueiredo LFP - Hypertonic-hyperoncotic solutions. Baillieres Clin Anaesth, 1997;2:143-161.

49. Nakayama S, Sibley L, Gunther R et al - Small-volume resuscitation with hypertonic saline $(2,400 \mathrm{mOsm} / \mathrm{liter})$ during hemorrhagic shock. Circ Shock, 1984;13:149-159.

50. Nakayama S, Kramer GC, Carlsen RC et al - Infusion of very hypertonic saline to bled rats: membrane potentials and fluid shifts. J Surg Res, 1985;38:180-186.

51. Matteucci MJ, Wisner DH, Gunther RA et al - Effects of hypertonic and isotonic fluid infusion on the flash evoked potential in rats: hemorrhage, resuscitation, and hypernatremia. J Trauma, 1993;34:1-7.

52. Mazzoni MC, Borgstrom P, Arfors KE et al - Dynamic fluid redistribution in hyperosmotic resuscitation of hypovolemic hemorrhage. Am J Physiol, 1988;255:H629-H637.

53. Mazzoni MC, Lundgren E, Arfors KE et al - Volume changes of an endothelial monolayer on exposure to anisotonic media. J Cell Res, 1989;140:272-280.

54. Hunter M, Lee $\mathrm{J}$ - Determination of fluid extraction and osmotic conductance sigma $\mathrm{K}$ in the lung with hypertonic $\mathrm{NaCl}$ infusion. II. Experiments. Microvasc Res, 1992;44:319-333.

55. Scalia SV, Taheri PA, Force S et al - Mesenteric microcirculatory changes in nonlethal hemorrhagic shock: the role of resuscitation with balanced electrolyte or hypertonic saline/dextran. J Trauma, 1992;33:321-325.

56. Bauer M, Marzi I, Ziegenfuss T et al - Comparative effects of crystalloid and small volume hypertonic hyperoncotic fluid resuscitation on hepatic microcirculation after hemorrhagic shock. Circ Shock, 1993;40:187-193.

57. Shackford SR, Fortlage DA, Peters RM et al - Serum osmolar and electrolyte changes associated with large infusions of hypertonic sodium lactate for intravascular volume expansion of patients undergoing aortic reconstruction. Surg Gynecol Obstet, 1987;164:127-136.

58. Shackford SR, Norton $\mathrm{CH}$, Todd MM - Renal, cerebral, and pulmonary effects of hypertonic resuscitation in a porcine model of hemorrhagic shock. Surgery, 1988;104:553-560.

59. Dubick MA, Wade CE - A review of the efficacy and safety of $7.5 \% \mathrm{NaCl} / 6 \%$ dextran 70 in experimental animals and in humans. J Trauma, 1994;36:323-330. 


\section{RESUMEN}

Meletti JFA, Braz JRC, Módolo NSP - Efectos Hemodinámicos y Metabólicos Inmediatos Determinados por las Soluciones de Cloruro de Sodio a $7,5 \%$ y de su Asociación con Dextran 70 a $6 \%$ en la Reanimación del Choque Hemorrágico. Estudio Experimental en perros.

JUSTIFICATIVA Y OBJETIVOS: El dextran, asociado a la solución hipertónica de cloruro sodio a 7,5\% presenta efectos hemodinámicos benéficos en el control prolongado de la reanimación en el choque hemorrágico. El objetivo de este estudio fue verificar si la asociación del dextran a la solución de cloruro de sodio a $7,5 \%$ presentaría ventajas en la evaluación inmediata de los parámetros hemodinámicos y metabólicos en reanimación de modelo de choque hemorrágico controlado en perros.

MÉTODO: Se estudiaron 16 perros sometidos a la hemorragia controlada hasta que la presión arterial promedio alcanzase $40 \mathrm{mmHg}$ y permaneciese asi hasta 30 minutos. Ellos fueron divididos en G1 administración de $\mathrm{NaCl}$ a 7,5\% y G2 administración $\mathrm{NaCl}$ a 7,5\% combinada con dextran 70 a $6 \%$, en un volumen de $4 \mathrm{~mL} . \mathrm{kg}^{-1}$, durante tres minutos. Se evaluaron los parámetros hemodinámicos y metabólicos. Se tuvo en cuenta cuatro momentos de estudio: (M1) 10 minutos después de la preparación quirúrgica, (M2) obtenido en la mitad de la fase de choque, (M3) obtenido dos minutos después del final de la administración de las soluciones, (M4) 30 minutos después del inicio de la reanimación.

RESULTADOS: Después de la reanimación, no hubo diferencia significativa de los valores de la FC, PAM, PCP y IRVS. EI G2 presentó valores mayores del IC en M4. Los valores de la $\mathrm{SvO}_{2}$ fueron menores en el G1, final del experimento. La $C_{(a-v)} \mathrm{O}_{2}$ fue mayor en el G1 en los momentos M3 y M4. Los valores del $\mathrm{VO}_{2}$ aumentaron en los dos grupos en M4 y los valores del lactato plasmático aumentaron progresivamente hasta llegar a M3 disminuyendo en M4. Hubo un aumento de los valores del en la plasmático y una reducción del hematócrito en los dos grupos.

CONCLUSIONES: EI G2 mostró un mejor desempeño hemodinámico principalmente después de 30 minutos del inicio de la reanimación. Se observó también, una mayor expansión plasmática y una mejor perfusión de tejido en la asociación del dextran con $\mathrm{NaCl}$ a $7,5 \%$. 